TRANSACTIONS OF THE

AMERICAN MATHEMATICAL SOCIETY

Volume 354, Number 12, Pages 5005-5026

S 0002-9947(02)03098-2

Article electronically published on August 1, 2002

\title{
EQUILIBRIUM EXISTENCE AND TOPOLOGY IN SOME REPEATED GAMES WITH INCOMPLETE INFORMATION
}

\author{
ROBERT S. SIMON, STANISŁAW SPIEŻ, AND HENRYK TORUŃCZYK
}

\begin{abstract}
This article proves the existence of an equilibrium in any infinitely repeated, un-discounted two-person game of incomplete information on one side where the uninformed player must base his behavior strategy on statedependent information generated stochastically by the moves of the players and the informed player is capable of sending nonrevealing signals.

This extends our earlier result stating that an equilibrium exists if additionally the information is standard. The proof depends on applying new topological properties of set-valued mappings. Given a set-valued mapping $F$ on a compact convex set $P \subset \mathbb{R}^{n}$, we give further conditions which imply that every point $p_{0} \in P$ belongs to the convex hull of a finite subset $P_{0}$ of the domain of $F$ satisfying $\bigcap_{x \in P_{0}} F(x) \neq \emptyset$.
\end{abstract}

\section{Introduction}

The motivation for considering results of this paper comes from game theory, and specifically from two-person infinitely repeated games of incomplete information on one side. With such a game, there is an infinite sequence of playing stages and the relationship between the moves and the payoffs, determined by nature at the start of the game but not necessarily known by both players, does not change in the course of the game. There are finitely many different possible relationships between the moves and the payoffs and they are called "states of nature". "Incomplete information on one side" means that one player, the "informed player", knows which state of nature is being played and both players know the probability of the given state to occur; moreover, the informed player knows everything his opponent knows.

In [SST] the existence of equilibria was established in such games with standard information. (For only two states of nature, the result was proven by Sorin [So].)

Received by the editors August 14, 2000 and, in revised form, May 10, 2002.

2000 Mathematics Subject Classification. Primary 55M20, 91A20; Secondary 54C60, 52A20, 91A05, 91A 10 .

The first named author wishes to thank Christof Wehrsig of the Sociology Department of the University of Bielefeld for introducing him to game theory. The research of this author was supported by the German Science Foundation (Deutsche Forschungsgemeinschaft), the Institute of Mathematical Economics (Bielefeld), the Institute of Mathematical Stochastics (Goettingen), the Center for Rationality and Interactive Decision Theory (Jerusalem), the Department of Mathematics of the Hebrew University (Jerusalem), and the Edmund Landau Center for Research in Mathematical Analysis (Jerusalem), sponsored by the Minerva Foundation (Germany). The Stefan Banach International Center at the Institute of Mathematics of the Polish Academy of Sciences enabled for meetings of the three authors while the paper was in preparation.

(C)2002 American Mathematical Society 
By standard information we mean that there is perfect monitoring (both players have perfect knowledge of all the past moves) and that the uninformed player's perception of these moves is independent of the state of nature. Due to perfect monitoring, the uninformed player may of course draw inferences concerning the state of nature from the sequence of moves of his opponent.

The purpose of this paper is to show that the state independence of information and the perfect monitoring can be discarded, provided the informed player can send nonrevealing signals (distinctly perceivable messages, the perception of which is independent of the state of nature). With other assumptions on the form of the game left intact, we show it still has an equilibrium.

In fact, we establish the existence of equilibria for games that are slightly more general than those of incomplete information on one side, namely when the player uninformed about the state of nature can obtain information only from perception that is determined stochastically by the moves of the players and the state. (We keep the assumption that the player who knows the state is able to send nonrevealing signals.) Here one must be careful, because there may be no equilibrium if the player knowing the state has no influence on or knowledge of some information received by the other player. Such is the case with zero-sum games of incomplete information on "one and a half sides". There, Player One knows the state of nature and the initial distribution on the states of nature, but does not know some additional revelation from Nature to Player Two of a posterior probability (see Sorin and Zamir $[\mathrm{SZ}]$ ).

There are a few steps in the proof, related to different ideas, and they can be separated as follows.

1. First, we show that the game can be split into several games which have state-independent information and correspond to certain subsets $L$ of the set $K$ of states of nature. The desired result follows once we are able to find equilibria for the arising sub-games that can be pasted together into an equilibrium of the original game. Moreover, for each of the subgames, there exists a class of strategy pairs, which we call "joint plans"; they are a revision, due to J. Renault, of a concept introduced by R. Aumann, M. Maschler, and R. Stearns, see [AM], with regard to the games with standard information. Out of these joint plans, we define a class of strategy pairs for the original game.

2. This step goes back to an idea from our previous paper [SST]. To each of our strategies for the game with probability distribution on $K$ equal to $p$ we associate a set $F(p)$ devised so that its nonemptiness implies that we reached an equilibrium. (See also 4 below.) $F(p)$ is a subset of the cube $I^{K}$ and is defined in terms of some conditions on the payoffs of the game. Identifying each probability distribution on $K$ with a point in the simplex $\Delta(K)$ formally spanned by $K$, we know in turn from SST that $F(q) \neq \emptyset$ for all $q \in \Delta(K)$ provided the correspondence $F: p \mapsto F(p)$, when considered as a subset of $\Delta(K) \times I^{K}$, has a certain (co-)homological property, named property $\mathcal{S}$ in the present paper. A similar situation exists with respect to the correspondences $F_{L} \subset \Delta(L) \times I^{L}$ associated with the subgames, with $L$ running over an appropriate family of subsets of $K$. By extending one of the main results from [SST] in an essential way, we show that the $F_{L}$ 's do have the property $\mathcal{S}$.

3 . The next step is to relate the correspondence $F$ to the $F_{L}$ 's. The correspondences $F_{L}$ and $F$ share the essential feature of being "saturated", meaning (for $F$ ) that whenever $y \in F(p)$ and $p_{i}=0$ for some $i \in K$, then $z \in F(p)$ for all $z \in I^{K}$ that differ from $y$ in the $i$-th coordinate only and satisfy $z_{i} \geq y_{i}$. We show that for 
a relevant class of saturated correspondences $\Delta(K) \rightarrow I^{K}$, property $\mathcal{S}$ is equivalent to the requirement that its image separates the cube between a certain vertex and the union of faces disjoint from it. This allows us to use the basic properties of separating sets to show that the property $\mathcal{S}$ of the correspondence $F$ follows from that of the $F_{L}$ 's.

4. The final step is to show that, given the nonemptiness of the set $F(p)$, the joint plans defining it do indeed generate equilibria. Here we depend largely on work of Renault [Re], who proved equilibrium existence when the uninformed player does have state-independent perception. In fact, we refer to his paper concerning the complex arguments involving the statistics of such games, and we make only a minimal revision of Renault's construction of behavior strategies from "joint plans".

The paper is not organized according to this outline. We cumulate our topological results, given in an abstract framework of saturated correspondences, in Section 2. There, steps 2 and 3 are covered. In Section 3 games of incomplete information are discussed, and we deal with steps 1 and 4 .

Although the main result belongs to game theory, the topological analysis plays a very essential role in the paper. Expressed in terms of correspondences, a version of one of the two results needed says the following. Assume for a certain cover $\mathcal{L}$ of a finite set $K$ that we are given a family $\left\{F_{L}: \Delta(L) \rightarrow[0,1]^{L}\right\}_{L \in \mathcal{L}}$ of saturated correspondences with property $\mathcal{S}$, such that the image of $F_{L}$ lies in a closed and convex subset $C_{L} \subset[0,1]^{L}$ containing $(1,1, \ldots, 1)$. Let $C:=\bigcap_{L \in \mathcal{L}} C_{L} \times[0,1]^{K \backslash L} \subset$ $[0,1]^{K}$. Then, for every point $p \in \Delta(K)$, there exist finitely many sets $L_{1}, \ldots, L_{s} \in$ $\mathcal{L}$ and points $p_{i} \in \Delta\left(L_{i}\right) \subset \Delta(K), i=1, \ldots, s$, such that $p \in \operatorname{conv}\left\{p_{i}\right\}_{i=1}^{s}$ and, with $\pi_{i}: I^{K} \rightarrow[0,1]^{L_{i}}$ denoting the projection, we have $\pi_{i}(y) \in F_{L_{i}}\left(p_{i}\right)$ for some $y \in C$ and $i=1, \ldots, s$. (See Corollary 2 in Section 2.) As indicated earlier, the proof is a manifestation of the fact that certain saturated correspondences have property $\mathcal{S}$ if and only if their image disconnects the target cube in a certain way. In addition to showing this equivalence, we formalize the property $\mathcal{S}$ (which in [SST] was used only implicitly) and establish other results that allow for its verification. We hope that these results concerning the topological aspects of infinitely repeated games and their associated correspondences will find more applications.

Basic notation (common to both sections). Spaces are assumed metric and we write $\operatorname{cl} C$ and $\bar{C}$ for the closure of a set $C$, int $C$ for the interior of $C$ and $\delta C$ for the boundary of the interior of $C$. If $K$ is a finite set, then by $\mathbb{R}^{K}$ we denote the Euclidean space of all functions $K \rightarrow \mathbb{R}$, equipped with the standard inner product, and by $\Delta(K)$ the simplex in $\mathbb{R}^{K}$ :

$$
\Delta(K):=\left\{y \in \mathbb{R}^{K}: y_{k} \geq 0 \text { for all } k \in K \text { and } \sum_{k} y_{k}=1\right\} .
$$

If $y \in \mathbb{R}^{K}$ and $L \subset K$, then $y^{L}$ denotes the projection of $y$ to $\mathbb{R}^{L}$; we may also (and often shall) identify $\Delta(L)$ with $\left\{y \in \Delta(K): y^{K \backslash L}=0\right\}$ and hence consider $\Delta(L)$ as a subset of $\Delta(K)$.

The $k$-th coordinate of a point $y \in \mathbb{R}^{K}$ is denoted both $y_{k}$ (mainly in Section 2) and $y^{k}$ (in Section 3, where additional indices are needed).

Given $a: \Delta(K) \rightarrow \mathbb{R}$ we denote by $\operatorname{cav}(a): \Delta(K) \rightarrow \mathbb{R}$ the concave envelope of $a$, defined by the property that the set $\{(x, t) \in \Delta(K) \times \mathbb{R}: t \leq \operatorname{cav}(a)(x)\}$ is the convex hull of $\{(x, t) \in \Delta(K) \times \mathbb{R}: t \leq a(x)\}$. 


\section{TOPOLOGICAL PROPERTIES OF CORRESPONDENCES}

2.1. Correspondences and their levelwise convexification. By a correspondence $F: X \rightarrow Y$ we mean any subset of $X \times Y$ such that its projection to $X$ is a proper mapping (i.e., is closed and has compact point-inverses). Thus, the same correspondence may also be denoted as $F \subset X \times Y$; the notation $F: X \rightarrow Y$, however, will most often be used as one that openly displays the nonsymmetric role that the spaces $X$ and $Y$ play here. The image of $F$ under the coordinate-switching map is denoted $F^{-1}$. It is a correspondence from $Y$ to $X$ provided the projection $F \rightarrow Y$ is proper.

If $X_{0}$ is a subset of $X$, then $F \cap\left(X_{0} \times Y\right)$ is called the restriction of $F$ to $X_{0}$ and denoted $F \mid X_{0}$. This notation applies also to single-valued correspondences $X \rightarrow Y$, which we call mappings.

For a correspondence $F: X \rightarrow Y$, the image of the set $F \mid X_{0}$ under the projection to $Y$ is denoted by $F\left(X_{0}\right)$ and called the image of $X_{0}$ under $F$. We also write

$\operatorname{im}(F):=F(X), F(x):=F(\{x\})$ for $x \in X$ and $\operatorname{dom}(F):=\{x \in X: F(x) \neq \emptyset\}$.

If $F: X \rightarrow Y$ is a correspondence, it is not assumed a priori that $F(x) \neq \emptyset$ for all (or even for some) $x \in X$. In fact, the demonstration of the nonemptiness of some of the sets $F(x)$ constitutes the main goal of this section.

In most of our considerations, $X$ is a subset of a Euclidean space $E$ in which a certain simplex $\Delta$ is selected along with a retraction $r: E \rightarrow \Delta$. We may then consider an operation on compact subsets $A$ of $E$ defined by the formula

$$
\operatorname{co}(A):=\operatorname{conv}(r(A)) \cup\{\lambda a+(1-\lambda) r(a): a \in A, \lambda \in[0,1]\} .
$$

(If $A \subset \Delta$, this reduces to taking the convex hull of $A$.) This operation may be applied levelwise to the correspondence $F: X \rightarrow Y$ to give a new correspondence $c F: E \rightarrow Y$ defined by

$$
c F:=\bigcup_{y \in Y} \operatorname{co}\left(F^{-1}(y)\right) \times\{y\}
$$

Thus,

$$
\begin{aligned}
& c F(p) \\
& =\left\{y \in Y: p \in \operatorname{co}\left(X_{0}\right) \text { for some finite set } X_{0} \subset E \text { such that } y \in \bigcap_{x \in X_{0}} F(x)\right\} .
\end{aligned}
$$

We say that $c F$ is obtained by convexifying $F$ level-wise.

The problem faced in our game-theoretic applications is to establish that, for a given correspondence $F: \Delta \rightarrow Y$, we have $\operatorname{dom}(c F) \supset \Delta$. The importance of such a conclusion is that it allows interpreting the nonemptiness of $(c F)(p)$, for a given point $p \in \Delta$, in terms of the existence of solutions to systems of inequalities encountered in game theory. The approach of handling this problem, developed in SST, depended on the use of certain homology elements carried by boundaries of open subsets of Euclidean spaces. Below we give an equivalent definition of these elements and prove those of their properties that are needed here.

2.2. Fundamental classes of boundaries of open subsets of $\mathbb{R}^{n}$. In what follows, $\tilde{H}$ denotes the reduced Čech homology functor with coefficients in a compact abelian group, cf. [ES] §IX.7]. $\tilde{H}(X, Y)$ is considered as a graded group, i.e., as 
a direct sum of the $\tilde{H}_{n}(X, Y)$ 's, and a specific group $\tilde{H}_{n}(X, Y)$ is isolated only if needed. As is usual, $\tilde{H}(X)$ is identified with $\tilde{H}(X, \emptyset)$.

Let $A \supset B$ and $A^{\prime} \supset B^{\prime}$ be compact pairs such that $A \subset A^{\prime}$ and $B \supset A \cap B^{\prime} \cup \delta A$. (We consider $A^{\prime}$ as the ambient space in which $\delta A$ is taken.) We have canonical homomorphisms

$$
\tilde{H}\left(A^{\prime}, B^{\prime}\right) \rightarrow \tilde{H}\left(A^{\prime}, B \cup\left(A^{\prime} \backslash \operatorname{int} A\right)\right) \rightarrow \tilde{H}(A, B),
$$

the first of which is inclusion-induced and the second of which is the composition of the excision of $A^{\prime} \backslash \operatorname{cl}(\operatorname{int} A)$ and the inclusion into $(A, B)$. (This excision is legitimate, by the continuity of Cech homology; see [ES, $\S$ X.3 and $\S$ X.5].) The arising homomorphism $\tilde{H}\left(A^{\prime}, B^{\prime}\right) \rightarrow \tilde{H}(A, B)$ will be called restriction and denoted $z \mapsto z \mid(A, B)$.

Remark 1. Let $C_{0}, C_{1}, C_{2}$ be compact subsets of a space $X$.

a) If $C_{1} \cap C_{2} \subset C_{0} \subset C_{1} \cup C_{2}$ then, by the Mayer-Vietoris exact sequence,

$$
\begin{gathered}
z \mapsto z\left|\left(C_{1}, C_{0} \cap C_{1}\right) \oplus z\right|\left(C_{2}, C_{0} \cap C_{2}\right) \quad \text { establishes an isomorphism } \\
\tilde{H}\left(C_{1} \cup C_{2}, C_{0}\right) \rightarrow h\left(C_{1}, C_{0} \cap C_{1}\right) \oplus \tilde{H}\left(C_{2}, C_{0} \cap C_{2}\right),
\end{gathered}
$$

the inverse of which is the sum of two inclusion-induced homomorphisms.

b) Assume $C_{0}=C_{1} \cap C_{2}$ and $z_{i} \in \tilde{H}\left(C_{i}, C_{0}\right)$ for $i=1,2$. Then, a $z \in \tilde{H}\left(C_{1} \cup C_{2}\right)$ such that $z \mid\left(C_{1}, C_{0}\right)=z_{1}$ and $z \mid\left(C_{2}, C_{0}\right)=z_{2}$ exists iff $\partial z_{1}+\partial z_{2}=0$, where $\partial$ denotes the boundary operator of the corresponding pair (by the same reason).

c) If $z \in \tilde{H}\left(C_{1} \cup C_{2}\right)$ and $z \mid\left(C_{1}, C_{1} \cap C_{2}\right)=0$, then $z \in \operatorname{im}\left(\tilde{H}\left(C_{2} \hookrightarrow C_{1} \cup C_{2}\right)\right)$ (which follows using excision and the exact sequence of the pair $\left(C_{1} \cup C_{2}, C_{2}\right)$ ).

Now consider $\mathbb{R}^{n}$ as a subset of the sphere $S^{n}=\mathbb{R}^{n} \cup\{\infty\}$, oriented by a certain $\omega \in \tilde{H}_{n}\left(S^{n}\right)$. Let $U$ be an open bounded set in $\mathbb{R}^{n}$. Taking $A^{\prime}=S^{n}$ and $B^{\prime}=\emptyset$, we denote by $[\delta U] \in \tilde{H}(\delta U)$ the image of $\omega \mid(\bar{U}, \delta U) \in \tilde{H}(\bar{U}, \delta U)$ under the boundary operator.

It needs to be stressed that if $\delta U$ is the boundary of several bounded open sets in $\mathbb{R}^{n}$, then the class $[\delta U]$ depends on which of them is being taken for $U$. In the most intuitive and still important case, however, when $\delta U$ is an $(n-1)$-dimensional compact closed PL-manifold, the choice of $U$ is unique and $[\delta U]$ can be shown to be the homology class of the cycle induced by this manifold (oriented according to $\omega$ ); this motivates our notation.

If $C \subset \mathbb{R}^{n}$ is a compact set, then we let $[\delta C]:=[\delta(\operatorname{int} C)] \in \tilde{H}(\delta C)$. We note that $[\delta C]$ is the image of $\omega \mid(C, \delta C) \in \tilde{H}(C, \delta C)$ under the boundary operator, since $\operatorname{dim}(C \backslash \operatorname{int} C)<n$.

Lemma 1. Let $C_{1}, C_{2}, C_{3}$ be compact sets in $\mathbb{R}^{n}$.

a) Suppose $C_{1} \subset C_{2} \subset C_{3}$ and let $z \in \tilde{H}\left(C_{3}, \delta C_{2}\right)$ be such that its image $\partial z$ under the boundary operator of the pair $\left(C_{3}, \delta C_{2}\right)$ is equal to $\left[\delta C_{2}\right]$. Then, $z\left|\left(C_{2}, \delta C_{2}\right)=\omega\right|\left(C_{2}, \delta C_{2}\right)$ and the image of $z \mid\left(C_{1}, \delta C_{1}\right)$ under the boundary operator of the pair $\left(C_{1}, \delta C_{1}\right)$ is $\left[\delta C_{1}\right]$.

b) If int $C_{1} \cap C_{2}=\emptyset$, then $\left[\delta\left(C_{1} \cup C_{2}\right)\right]^{\prime}=\left[\delta C_{1}\right]^{\prime}+\left[\delta C_{2}\right]^{\prime}$, where' denotes taking the image in $\tilde{H}\left(\delta C_{1} \cup \delta C_{2}\right)$ of the corresponding homology class.

c) Suppose either int $C_{1} \cap C_{2}=\emptyset$ or $\operatorname{int} C_{1} \subset C_{2}$. With $A_{0}:=\delta C_{1} \cap \delta C_{2}$ and $B_{0}:=A_{0} \cap \delta\left(C_{1} \cup C_{2}\right)$, we have $\left[\delta C_{1}\right]\left|\left(A_{0}, B_{0}\right)= \pm\left[\delta C_{2}\right]\right|\left(A_{0}, B_{0}\right)$, where the sign is positive if int $C_{1} \subset C_{2}$ and is negative if int $C_{1} \cap C_{2}=\emptyset$. 
d) If $\delta C_{1} \subset C_{2}$, then the condition $\operatorname{int} C_{1} \subset C_{2}$ is equivalent to $\tilde{H}\left(\delta C_{1} \hookrightarrow\right.$ $\left.C_{2}\right)\left[\delta C_{1}\right]=0$.

Proof. a). Let $z_{0}$ be the image of $\omega \mid\left(C_{2}, \delta C_{2}\right)$ under the inclusion-induced homomorphism $j: \tilde{H}\left(C_{2}, \delta A_{2}\right) \rightarrow \tilde{H}\left(C_{3}, \delta C_{2}\right)$. Then, $\partial z_{0}=\left[\delta C_{2}\right]=\partial z$. Thus $z-z_{0}$ is in the image of $\tilde{H}_{n}\left(C_{3}\right)$ in $\tilde{H}_{n}\left(C_{3}, \delta C_{2}\right)$ and so is equal to 0 , since the $n$-th homology of a compact set in $\mathbb{R}^{n}$ vanishes. Since $j$ composed with restriction to $\left(C_{2}, \delta C_{2}\right)$ is the identity, we get $z\left|\left(C_{2}, \delta C_{2}\right)=z_{0}\right|\left(C_{2}, \delta C_{2}\right)=\omega \mid\left(C_{2}, \delta C_{2}\right)$. The second claim now follows from the fact that $\left(\omega \mid\left(C_{2}, \delta C_{2}\right)\right)\left|\left(C_{1}, \delta C_{1}\right)=\omega\right|\left(C_{1}, \delta C_{1}\right)$.

b). It is easy to see that indeed $\delta\left(C_{1} \cup C_{2}\right) \subset \delta C_{1} \cup \delta C_{2}$. Assuming, as we may, that $C_{1}$ and $C_{2}$ are closures of open sets, let $y:=\omega \mid\left(C_{1} \cup C_{2}, \delta\left(C_{1} \cup C_{2}\right)\right)$ and $z_{i}:=\omega \mid\left(C_{i}, \delta C_{i}\right)$. Then, by (3), the images of $z_{i}$ in $\tilde{H}\left(C_{1} \cup C_{2}, \delta C_{1} \cup \delta C_{2}\right)$ add up to the image of $y$ in that group. Taking boundaries, we get the conclusion.

c). By replacing $C_{2}$ by $C_{2}^{\prime}:=S^{n} \backslash \operatorname{int} C_{2}$ and $\infty$ by a point of $\operatorname{int} C_{2}$, we notice that it suffices to consider the case when int $C_{1} \cap C_{2}=\emptyset$; we thus assume the latter. By b), $\left[\delta C_{1}\right]^{\prime}+\left[\delta C_{2}\right]^{\prime}$ and $\left[\delta\left(C_{1} \cup C_{2}\right)\right]^{\prime}$ have the same restrictions to $\left(A_{0}, B_{0}\right)$. Moreover, $A_{0} \backslash B_{0}$ is disjoint from $\delta\left(C_{1} \cup C_{2}\right)$ whence $\left[\delta\left(C_{1} \cup C_{2}\right)\right]^{\prime} \mid\left(A_{0}, B_{0}\right)=0$. So $\left(\left[\delta C_{1}\right]^{\prime}+\left[\delta C_{2}\right]^{\prime}\right) \mid\left(A_{0}, B_{0}\right)=0$.

d). We have $\tilde{H}\left(\delta C_{1} \hookrightarrow \operatorname{cl}\left(\operatorname{int} C_{1}\right)\right)\left[\delta C_{1}\right]=0$ by the exact sequence of a pair. Thus, $\tilde{H}\left(\delta C_{1} \hookrightarrow C_{2}\right)\left[\delta C_{1}\right]=0$ if $C_{2} \supset \operatorname{int} C_{1}$.

Now assume that, conversely, $\tilde{H}\left(\delta C_{1} \hookrightarrow C_{2}\right)\left[\delta C_{1}\right]=0$ and let $Q=[-M, M]^{n}$ be a cube such that $C_{1} \cup C_{2} \subset \operatorname{int} Q$. With $C:=Q \backslash \operatorname{int} C_{1}$, we get from b) that $[\delta C]=[\delta Q]^{\prime}-\left[\delta C_{1}\right]^{\prime}$, where ' denotes taking images in $\tilde{H}(\delta C)$. The image of $[\delta C]$ in $\tilde{H}(C)$ being 0 , it follows that $[\delta Q]$ and $\left[\delta C_{1}\right]$ have the same image in $\tilde{H}\left(C \cup C_{2}\right)$. Hence the image of $[\delta Q]$ is 0 , which can happen only if $Q \subset C \cup C_{2}$. Thus int $C_{1} \subset C_{2}$.

\subsection{Spanning homological elements.}

Definition. A correspondence $F: X \rightarrow Y$ is acyclic-valued if each set $F(x), x \in$ $X$, is acyclic, i.e., is nonempty and satisfies $\tilde{H}(F(x))=\tilde{H}$ (\{point\}); similarly, if $Y=\mathbb{R}^{n}$, we say that $F$ is convex-valued if each set $F(x)$ is nonempty and convex. We call a mapping $f: X \rightarrow Y$ acyclic if the correspondence $f^{-1}: \operatorname{im}(f) \rightarrow X$ is acyclic-valued.

We now state a result from $[\underline{\mathrm{SST}}]$ and isolate a property established in it 1

Theorem 0. Let $E$ be an $n$-dimensional affine space, $U$ be its open bounded subset, and $F: \bar{U} \rightarrow Y$ be an acyclic-valued correspondence. If $\operatorname{dim} F(U)<n$, then an element of $\tilde{H}_{n-1}(F \mid \delta U)$ is mapped to $[\delta U]$ by $\tilde{H}(F \mid \delta U \rightarrow \delta U)$ and to 0 by $\tilde{H}(F \mid \delta U \hookrightarrow c(F \mid \delta U))$.

Definition. Let $E$ be as above. A correspondence $F: E \rightarrow Y$ is said to have property $\mathcal{S}$ for an open bounded set $U \subset E$ if $F$ is a compact subset of $E \times Y$ and there exists an element $z \in \tilde{H}_{n-1}(F \mid \delta U)$ whose images under $\tilde{H}(F \mid \delta U \rightarrow \delta U)$ and under $\tilde{H}(F \mid \delta U \hookrightarrow F)$ are $[\delta U]$ and 0 , respectively. If, moreover, for a given compact set $Z \subset F \mid \delta U$ one may take $z$ in the image of $\tilde{H}(Z \hookrightarrow F \mid \delta U)$, then we say that $Z$ witnesses the property $\mathcal{S}$ for $U$. We call $z$ a spanning element for

\footnotetext{
${ }^{1}$ Strictly speaking, Theorem 1 of [SST] covers the case where $U=\operatorname{int} \bar{U}$, but its proof establishes the full result.
} 
$U$ because if it exists (that is, if $F$ has property $\mathcal{S}$ for $U$ ), then $\operatorname{dom}(F) \supset U$ by Lemma $1(\mathrm{~d})$.

We say that the correspondence $F$ has property $\mathcal{S}$ for a compact set $C \subset E$ if $F$ has property $S$ for the interior of $C$.

It turns out that property $\mathcal{S}$ is hereditary and continuous and can under some assumptions be pasted together, as explained below:

Lemma 2. Let $F: E \rightarrow Y$ be a compact correspondence and $C \subset E$ be a compact set. Then:

a) If $F$ has property $\mathcal{S}$ for a compact set $D \supset C$, then $F \mid C$ has it for $C$.

b) $F$ has property $\mathcal{S}$ for $C$ provided that for every neighborhood $U$ of $\delta C$ in $E$ and for every neighborhood $V$ of $F$ in $E \times Y$, there exists a correspondence $G: E \rightarrow Y$ such that $G \subset V$ and $G$ has property $\mathcal{S}$ for a compact set $D$ satisfying $\delta D \subset U$ and $D \subset C \cup U$.

In particular, if each of the correspondences $F_{i}: E \rightarrow Y$ has property $\mathcal{S}$ for $C$ and $F_{1} \supset F_{2} \supset F_{3} \ldots$, then $\bigcap_{n} F_{n}$ has this property also.

c) Let $U_{1}$ and $U_{2}$ be disjoint open bounded subsets of $E$. If $G: \overline{U_{2}} \rightarrow Y$ is an acyclic-valued correspondence and the property $\mathcal{S}$ of $F$ for $U_{1}$ is being witnessed by a compact set $Z \subset F \mid \delta U_{1}$ such that $Z \mid \delta U_{2} \subset G$, then $F \cup G$ has property $\mathcal{S}$ for $\operatorname{cl}\left(U_{1} \cup U_{2}\right)$.

Proof. a). Let $x \in \tilde{H}(F, F \mid \delta D)$ satisfy $\tilde{H}(F \mid \delta D \rightarrow \delta D)(\partial x)=[\delta D]$ and let $y:=$ $x \mid(F|C, F| \delta C)$. By part a) of Lemma 1, applied to $\left(C_{1}, C_{2}, C_{3}\right)=(C, D, \operatorname{dom}(F))$ and $z$ equal to the image of $x$ under the projection-induced mapping, $\tilde{H}(F \mid \delta C \rightarrow$ $\delta C)(\partial y)=[\delta C]$.

b). The first part follows from the fact that the functor $\tilde{H}$ is continuous and takes values in compact groups. The second part follows from the first since the correspondences $F_{i} \mid C$ are compact and by a) have property $\mathcal{S}$ for $C$.

c). Let $z_{1} \in \tilde{H}(Z)$ be a spanning element for $U_{1}$ and $z_{0}:=z_{1} \mid\left(Z\left|A_{0}, Z\right| B_{0}\right)$, where we let

$$
\begin{aligned}
& A_{0}:=\delta U_{1} \cap \delta U_{2}, \quad D:=\overline{U_{1}} \cup \overline{U_{2}}, \\
& B_{0}:=A_{0} \cap \delta D, \quad B_{1}:=\delta U_{1} \cap \delta D, \quad B_{2}:=\delta U_{2} \cap \delta D .
\end{aligned}
$$

By Lemma $1(\mathrm{c})$ and Remark $1(\mathrm{~b})$, the image of $z_{0}$ in $\tilde{H}\left(A_{0}, B_{0}\right)$ equals $-\left[\delta U_{2}\right] \mid\left(A_{0}\right.$, $\left.B_{0}\right)$ and $\tilde{H}\left(Z \mid B_{0} \rightarrow B_{0}\right)\left(\partial z_{0}\right)=\partial\left(\left[\delta U_{2}\right] \mid\left(B_{2}, B_{0}\right)\right)$.

Claim. There exists a $z_{2} \in \tilde{H}\left(Z\left|A_{0} \cup G\right| B_{2}\right)$ such that $\tilde{H}\left(Z\left|A_{0} \cup G\right| B_{2} \rightarrow \delta U_{2}\right)\left(z_{2}\right)=$ $\left[\delta U_{2}\right]$ and $z_{2} \mid\left(Z\left|A_{0}, Z\right| B_{0}\right)=-z_{0}$.

Proof of the Claim. By Vietoris' theorem [Sp], there exists $\alpha \in \tilde{H}\left(G\left|B_{2}, G\right| B_{0}\right)$ such that $\tilde{H}\left(\left(G\left|B_{2}, G\right| B_{0}\right) \rightarrow\left(B_{2}, B_{0}\right)\right)(\alpha)=\left[\delta U_{2}\right] \mid\left(B_{2}, B_{0}\right)$. Then $\partial \alpha$ is the image of $\partial z_{0}$ in $\tilde{H}\left(G \mid B_{0}\right)$. By Remark $1(\mathrm{~b}), \alpha$ and $z_{0}$ induce the desired element $z_{2} \in$ $H\left(Z\left|A_{0} \cup G\right| B_{2}\right)$.

We return to the proof of part c). Let $z_{1}^{\prime}$ and $z_{2}^{\prime}$ be the images of $z_{1}$ and of $z_{2}$, respectively, in $\tilde{H}\left(Z \cup G \mid B_{2}\right)$. Then $x^{\prime}:=z_{1}^{\prime}+z_{2}^{\prime}$ has the property that $x^{\prime} \mid\left(Z\left|A_{0}, Z\right| B_{0}\right)=0$ and thus, by Remark $1(\mathrm{c})$, there exists an $x \in \tilde{H}\left(Z\left|B_{1} \cup G\right| B_{2}\right)$ such that the image of $x$ in $\tilde{H}\left(Z \cup G \mid B_{2}\right)$ is $x^{\prime}$. Since $z_{1}^{\prime}$ and $z_{2}^{\prime}$ map to zero in $\tilde{H}(F \cup G)$, so does $x$. 
It remains to show that $x$ maps to $[\delta D]$ under $\tilde{H}\left(Z\left|B_{1} \cup G\right| B_{2} \rightarrow B_{1} \cup B_{2}=\right.$ $\delta D)$. However, $[\delta D]$ and $x^{\prime}$ have, by part (b) of Lemma 1 , the same image in $\tilde{H}_{n-1}\left(\delta U_{1} \cup \delta U_{2}\right)$, and $\tilde{H}_{n-1}\left(\delta D \hookrightarrow \delta U_{1} \cup \delta U_{2}\right)$ is a monomorphism in view of $\operatorname{dim}\left(\delta U_{1} \cup \delta U_{2}\right)<n$. Our claim thus follows from the commutativity of a diagram induced by projections and inclusions.

Parts a) and b) imply:

Corollary 1. If a correspondence $F$ has property $\mathcal{S}$ for a compact set $C \subset E$, then so does any compact correspondence $G \subset E \times Y$ containing $F \mid \operatorname{int}(C)$.

\subsection{Two results about saturated correspondences.}

Definition. If $F: \Delta(L) \rightarrow Y$ is a correspondence and $Y \subset \mathbb{R}^{L}$, then by $F^{+}$we denote the correspondence $\Delta(L) \rightarrow Y$ defined by

$F^{+}(p):=\left\{y \in Y: \exists x \in F(p)\right.$ such that $x_{l} \leq y_{l}$ for all $l \in L$ and $x_{l}=y_{l}$ if $\left.p_{l}>0\right\}$.

We call $F^{+}$the $Y$-saturation of $F$. We say that $F$ is saturated if $F=F^{+}$.

In the following we write for brevity $\Delta$ for $\Delta(L)$, where $L$ is a fixed finite set. We also often identify vectors in $\mathbb{R}^{L}$ with the functions they induce on $\mathbb{R}^{L}$ by scalar multiplication. Thus for $A \subset \mathbb{R}^{L}$ and $\varphi \in \mathbb{R}^{L}$, it makes sense to denote by $\varphi \mid A$ the restricted function $A \rightarrow \mathbb{R}$, and $\varphi \mid A \geq a$ means for a given function $a: A \rightarrow \mathbb{R}$ that $\varphi \cdot q \geq a(q)$ for all $q \in A$.

Theorem 1. Let I be a closed segment in $\mathbb{R}$, let $F: \Delta \rightarrow I^{L}$ be a convex-valued correspondence, and let $a: \Delta \rightarrow I$ be a lower semi-continuous function such that

$$
a(q) \leq \sup \{\varphi \cdot q: \varphi \in F(p)\} \text { for all } p, q \in \Delta .
$$

With $F^{+}$denoting the $I^{L}$-saturation of $F$, the following correspondence $G: \Delta \rightarrow$ $\mathbb{R}^{L}$ has property $\mathcal{S}$ for $\Delta$ :

$$
G:=c\left(F^{+}\right) \cap\left(\Delta \times\left\{\varphi \in I^{L}: \varphi \mid \Delta \geq a\right\}\right) .
$$

Proof. By applying a shift in $\mathbb{R}$ and in $\mathbb{R}^{L}$, we may assume that $I=[-M, M]$ for some $M>0$. We divide the proof into 3 steps:

i) We first assume that $a$ is piecewise linear and the correspondence $F$ is continuous. As in [SST], p. 12, we consider a retraction $r: E \rightarrow \Delta$ and a mapping $u: E \rightarrow[0, \infty)^{L}$ defined by:

$E$ is the affine hull of $\Delta=\Delta(L)$ in $\mathbb{R}^{L}$,

$r(v)_{k}:=\max \left(v_{k}, 0\right) / \sum\left\{v_{l}: v_{l}>0\right\}$ and $u(v)_{k}:=\max \left(-v_{k}, 0\right)$ for all $k \in L$.

Using $r$ and $u$ we define:

- sets $F(v)$ for $v \in E \backslash \Delta$ by the formula $F(v):=F(r(v))+u(v)$,

- convex functions $b_{v}: \Delta \rightarrow \mathbb{R}$ and nonnegative numbers $z(v)$ for $v \in E$ by the formulas:

$$
\begin{gathered}
b_{v}(q):=\max _{\varphi \in F(v)} \varphi(q), \\
z(v):=\max _{q \in \Delta}\left(\operatorname{cav}(a)(q)-b_{v}(q)\right)=\max _{q \in \Delta} \min _{\varphi \in F(v)}(\operatorname{cav}(a)(q)-\varphi \cdot q),
\end{gathered}
$$


- correspondences $\Psi_{0}, \Psi: E \rightarrow \mathbb{R}^{L}$ by the formulas in which $e=(1, \ldots, 1) \in$ $\mathbb{R}^{K}$ :

$\Psi_{0}(v):=\{\varphi \in F(v): \varphi \mid \Delta \geq a\}, \quad \Psi(v):=\{\varphi \in F(v)+z(v) e: \varphi \mid \Delta \geq a\}$,

- an open set $U \subset E$ by the formula

$$
U:=\{v \in E: z(v)>0 \text { and }\|v\|<R\},
$$

where the value of $R>\sup \{\|v\|: v \in \Delta\}$ is to be specified later.

By the definition of $z(v)$ and by (4), each set $\Psi(v)$ is nonempty and convex and $a \leq \varphi \mid \Delta \leq b_{v}+z(v)$ for $\varphi \in \Psi(v)$. This implies as in [SST], p. 10, that $\Psi(U)$ is of dimension lower than that of $E$. (The needed inequality $a \leq b_{v}$ follows from (4).) Therefore, $\Psi \mid \delta U$ witnesses by Theorem 0 the property $\mathcal{S}$ (for $U$ ) of the correspondence $c(\Psi \mid \delta U)$.

Let us now consider the correspondence

$$
F^{\prime}:=c(\Psi \mid \delta U) \cup \Psi_{0} \mid(E \backslash U) .
$$

Clearly, $\Psi(p)=\Psi_{0}(p)$ if $p \in \delta U \cap \Delta$, while for $p \in E \backslash U$, the set $\Psi_{0}(p)$ is nonempty, compact and convex. Thus it follows from part (c) of Lemma 2 (applied with $U_{1}:=U, U_{2}:=\operatorname{int}(\Delta \backslash U)$ and $\left.Z:=\Psi \mid \delta U\right)$ that $F^{\prime}$ has property $\mathcal{S}$ for $\operatorname{cl}\left(U \cup U^{\prime}\right) \supset \Delta$, and from part (a) that $F^{\prime} \mid \Delta$ has property $S$ for $\Delta$. By Corollary 1 , it therefore remains to show that

(*) with an appropriate choice of $R$, we have $F^{\prime}(p) \subset G(p)$ for each $p \in \operatorname{int} \Delta$.

To this end, we fix $p \in \operatorname{int} \Delta$ and $\varphi \in F^{\prime}(p)$. If $\varphi \in \Psi_{0}(p)$, then $\varphi \in F(p)$ and $\varphi \mid \Delta \geq a$, and so $\varphi \in G(p)$; thus we assume that $\varphi \in c(\Psi \mid \delta U)(p)$. By definition, $\varphi \mid \Delta \geq a$ and there exists a finite set $V \subset \delta U$ such that $p \in \operatorname{conv}(r(V))$ and $\varphi \in F(r(v))+u(v)+z(v) e$ for all $v \in V$.

We use a trick from [SST, which is as follows. Since $p \in \operatorname{int} \Delta \cap \operatorname{conv}(r(V))$, for every $l \in L$ there exists a $w \in V$ such that $r(w)_{l}>0$, forcing $u(w)_{l}=0$ and $\varphi_{l} \in[-M, M]+0+[-M, M]=[-2 M, 2 M]$. Now, if $v$ is an arbitrary element of $V$, then $u(v) \in \varphi-z(v) e-F(r(v))$, whence $u(v)_{l} \leq 2 M+M+M=4 M$ and $v_{l} \geq-4 M$ for all $l \in L$, yielding $\|v\| \leq R_{0}:=\max \left\{\|y\|: y \in E \cap[-4 M, \infty)^{L}\right\}<\infty$. Since for $v \in \delta U$ we have $\|v\|=R$ or $z(v)=0$, it follows that taking $R>R_{0}$ forces $z(v)=0$ for all $v \in V$ and, hence, $\varphi \in F(r(v))+u(v) \subset F^{+}(r(v))$ for all $v \in V$. Also, the information that $z(w)=0$ improves the earlier estimate on $\varphi_{l}$ to $\varphi_{l} \in[-M, M]$ for all $l \in L$. Since $p \in \operatorname{conv}(r(V))$, this establishes that $\varphi \in c\left(F^{+}\right)(p)$ and completes the proof in the special case i) above.

ii) We now continue to assume that $F$ is continuous but drop the assumption that $a$ be PL. Then, $a^{\prime}$ defined by $a^{\prime}(q):=\inf _{p \in \Delta(L)} \sup _{y \in F(p)} y \cdot q$ is continuous and thus is a uniform limit of PL-functions $a_{n}: \Delta(L) \rightarrow \mathbb{R}$ such that $a_{1}<a_{2}<\ldots$. If we define correspondences $G_{n}$ using formula (5) with $a_{n}$ in place of $a$ and a correspondence $G^{\prime}$ using $a^{\prime}$ in place of $a$, then we easily see that $\bigcap_{n} G_{n}=G^{\prime} \subset G$. Since, as shown above, each of the correspondences $G_{n}$ has property $\mathcal{S}$ for $\Delta(L)$, we infer from part (b) of Lemma 2 that $G$ also does have this property.

iii) In the general case, we apply Lemma 2 of Simon $\mathrm{Si}$ for each $n \in \mathbb{N}$ to get a continuous correspondence $F_{n}: \Delta(L) \rightarrow \mathbb{R}^{L}$ such that $F_{n}$ contains $F$ and is a subset of the $\frac{1}{n}$-neighborhood of $F$ in $\Delta \times \mathbb{R}^{L}$. By taking intersections, we may request also that $F_{1} \supset F_{2} \supset \ldots$ The result again follows by a limit argument 
as above applied to correspondences $G_{n}$, which are defined using formula (5), this time with $F_{n}$ in place of $F$.

The above theorem is one of the two ingredients of our topological analysis. The other is the following result showing how property $\mathcal{S}$ can be improved (i.e., derived for a larger simplex) under certain operations on saturated correspondences.

Theorem 2. Let $I=[a, b]$ be a nontrivial closed segment in $\mathbb{R}, K$ a finite set and $\mathcal{L}$ a family of its non-void subsets such that $\bigcup \mathcal{L}=K$. Suppose for every $L \in \mathcal{L}$ there are given a saturated correspondence $F_{L}: \Delta(L) \rightarrow I^{L}$ with property $\mathcal{S}$ for $\Delta(L)$ and a closed convex subset $U_{L}$ of $I^{K}$ containing the point $(b, b, \ldots, b)$. With $\Delta(L)$ considered as a subset of $\Delta(K)$, set for $L \in \mathcal{L}$ :

$$
\tilde{F}_{L}:=F_{L} \times I^{K \backslash L}=\left\{(p, y) \in \Delta(L) \times I^{K}: y^{L} \in F_{L}(p)\right\} \subset \Delta(K) \times I^{K} .
$$

If $\operatorname{im}\left(\tilde{F}_{L}\right) \subset U_{L}$ for every $L \in \mathcal{L}$ and the correspondence $\Gamma: \Delta(K) \rightarrow I^{K}$ is defined as

$$
\Gamma:=\bigcup_{L \in \mathcal{L}} \tilde{F}_{L} \cap\left(\Delta(K) \times \bigcap_{L \in \mathcal{L}} U_{L}\right)
$$

then $c \Gamma$ has property $\mathcal{S}$ for $\Delta(K)$.

In particular, it follows that for every $p \in \Delta(K)$ we have $(c \Gamma)(p) \neq \emptyset$. We formulate this consequence explicitly in a form applied in the sequel:

Corollary 2. Under assumptions of Theorem 2, let $p \in \Delta(K)$ be given. Then, there exist: finitely many sets $L_{1}, \ldots, L_{s} \in \mathcal{L}$ and points $p_{i} \in \Delta\left(L_{i}\right) \subset \Delta(K), i=$ $1, \ldots, s$, and a point $\varphi \in \bigcap_{L \in \mathcal{L}} U_{L} \subset I^{K}$ such that the following conditions hold:

$$
p \in \operatorname{conv}\left\{p_{1}, \ldots, p_{s}\right\} \text { and } \varphi^{L_{i}} \in F_{L_{i}}\left(p_{i}\right) \text { for every } i=1, \ldots, s .
$$

The proof of Theorem 2 involves a relation between the property $\mathcal{S}$ of a correspondence $\Delta(K) \rightarrow \mathbb{R}^{K}$ and certain separation properties of its image. We establish this relation below. As is usual, we say that a closed set $S$ in a space $X$ separates $X$ between two sets $A, B \subset X$ if $X \backslash S$ is a union of two disjoint open sets, one of which contains $A$ and the other $B$.

The next lemma is known, but failing to find a reference we include a proof.

Lemma 3. Let $\Delta$ be an $n$-simplex in the affine $n$-space $E$. A correspondence $F$ : $\Delta \rightarrow(0,1)$ has property $\mathcal{S}$ for $\Delta$ if and only if the set $F$ separates $\Delta \times[0,1]$ between $\Delta \times\{0\}$ and $\Delta \times\{1\}$.

Proof. a) The "if" implication. Let $C$ be the component of $\Delta \times[0,1] \backslash F$ containing $\Delta \times\{0\}$ and let $D:=\delta C \cap(\delta \Delta \times[0,1]), D_{1}:=\Delta \times\{0\}$ and $D_{2}:=\operatorname{cl}\left(\delta C \backslash\left(D_{1} \cup D\right)\right)$. With $z_{i}:=[\delta C] \mid\left(D_{i}, D_{i} \cap D\right)$, we note that the image of $\partial z_{2} \in \tilde{H}\left(D_{2} \cap D\right)$ vanishes in $\tilde{H}\left(D_{2}\right)$ and so it does in $\tilde{H}(F)$ since $D_{2} \subset F$.

Let ' denote taking the image of homology classes in $\tilde{H}\left(D \cap\left(D_{1} \cup D_{2}\right)\right)$. Then $\partial z_{1}^{\prime}+\partial z_{2}^{\prime}=-\partial\left([\delta C] \mid\left(D, D \cap\left(D_{1} \cup D_{2}\right)\right)\right.$, by Remark 1(b) applied with $X=\delta C, C_{2}=$ $D$ and $C_{1}=D_{1} \cup D_{2}$. Thus the images in $\tilde{H}(\delta \Delta)$ of $\partial z_{1}$ and of $-\partial z_{2}$ under the projection $D \rightarrow \delta \Delta$ coincide and $-\partial z_{2}$ is a spanning element for $\Delta$.

b) The "only if" implication. Suppose $F$ fails to separate while having property $\mathcal{S}$. Then, there exists an arc $A$ connecting $\Delta \times\{0\}$ to $\Delta \times\{1\}$ and missing the set $F \cup \delta \Delta \times[0,1]$. Due to the assumed property $\mathcal{S}$ for $\Delta$, the cycle generated by $\delta \Delta \times\{0\}$ is null-homologous in $F \cup \delta \Delta \times[0,1]$. This contradicts the fact that this 
cycle is not null-homologous in the complement of the arc $A$ in $\Delta \times[0,1]$, for by the Mayer-Vietoris theorem, $\tilde{H}(\delta \Delta \times\{0\} \hookrightarrow \Delta \times[0,1] \backslash A)$ is an isomorphism.

Lemma 4. Let a correspondence $F: \Delta(L) \rightarrow \Delta(L) \times(0,1)$ satisfy the following conditions:

i) $F^{-1}: \operatorname{im}(F) \rightarrow \Delta(L)$ is acyclic-valued,

ii) $F^{-1}\left(\Delta\left(L_{0}\right) \times(0,1)\right) \subset \Delta\left(L_{0}\right)$ for each nonempty subset $L_{0}$ of $L$.

Then, in order for the image $\operatorname{im}(F)$ of $F$ to separate $\Delta(L) \times[0,1]$ between $\Delta(L) \times\{0\}$ and $\Delta(L) \times\{1\}$, it is necessary and sufficient that $F \cap(\delta \Delta(L) \times \delta \Delta(L) \times[0,1])$ witnesses the property $\mathcal{S}$ of $F$ for $\Delta(L)$.

Proof. We write $\Delta$ for $\Delta(L)$ and, when willing to distinguish between the factors of $\Delta \times \Delta \times[0,1]$, we denote the first by $\Delta_{1}$ and the second by $\Delta_{2}$. Consider $F^{\prime}:=\operatorname{im}(F)$ as a correspondence $\Delta \rightarrow(0,1)$ and denote by $\pi$ the projection of $F$ onto $F^{\prime}$ along $\Delta_{1}$, by $q$ the projection $\Delta \times[0,1] \rightarrow \Delta$ and by $p$ the projection $\Delta_{1} \times \Delta_{2} \times[0,1] \rightarrow \Delta_{1}$. By assumptions, $\pi$ is acyclic and for $L_{0} \subset L$ we have

for $x \in T:=F \cap\left(\Delta_{1} \times \delta \Delta_{2} \times[0,1]\right)$, if $q \pi(x) \in \Delta\left(L_{0}\right)$ then $p(x) \in \Delta\left(L_{0}\right)$.

Thus $T$ coincides with $F \cap\left(\delta \Delta_{1} \times \delta \Delta_{2} \times[0,1]\right)$ and $p$ and $q \pi$ are on $T$ homotopic as mappings into $\delta \Delta$ (use a straight line homotopy); they hence induce the same homomorphisms $\tilde{H}(T) \rightarrow \tilde{H}(\delta \Delta)$. Also, the acyclic map $\pi$ induces by Vietoris' theorem isomorphisms $\tilde{H}(F) \rightarrow \tilde{H}\left(F^{\prime}\right)$ and $\tilde{H}(T) \rightarrow \tilde{H}\left(F^{\prime} \mid \delta \Delta\right)$. From these facts and Lemma 3 , the conclusion readily follows.

Notation. Let $I=[a, b]$ be a nondegenerate closed segment. Given a finite set $K$, we denote by $v_{+}$and $v_{-}$the points $(b, \ldots, b)$ and $(a, \ldots, a)$ of the cube $I^{K}$, and for $L \subset K$ we let

$$
B(L):=\left\{y \in I^{K} \mid y_{k}=b \forall k \in K \backslash L\right\}
$$

and

$$
A(L):=B(L) \cap\left\{y \in I^{K} \mid y_{k}=a \text { for some } k \in L\right\} .
$$

Remark 2. Each set $B(L)$ is a geometric cone on $A(L)$ with vertex $v_{+}$(in particular, $I^{K}$ is a cone on $\left.A(K)\right)$; also, $A(K)$ is a cone on $\bigcup_{|L|<|K|} A(L)$ with vertex $v_{-}$. Consequently, there exists a homeomorphism $h$ of the cube $I^{K}$ onto the cone Cone $(\Delta(K))$ on $\Delta(K)$ that i) sends $v_{+}$to the vertex of the cone, ii) for each set $L \subset K$ sends the set $A(L)$ to the simplex $\Delta(L)$, and iii) is linear on any cone segment. In fact, to construct $h$, it suffices to use induction with respect to the number of elements of $K$ and to proceed as follows:

a) first, for every set $K^{\prime} \subset K$ with $\left|K^{\prime}\right|=|K|-1$, construct a homeomorphism $B\left(K^{\prime}\right) \rightarrow \operatorname{Cone}\left(\Delta\left(K^{\prime}\right)\right)$ having the desired properties and denote by $h_{0}$ the resulting homeomorphism $B \rightarrow \operatorname{Cone}(\delta \Delta(K))$, where $B:=\bigcup_{|L|<|K|} B(L)$;

b) get a homeomorphism $h_{1}: A(K) \rightarrow \Delta(K)$ by mapping each segment $\left[x, v_{-}\right]$, $x \in A(K) \cap B$, linearly to the segment from $h(x)$ to the center of $\Delta(K)$;

c) extend $h_{1}$ linearly over any cone segment by considering $I^{K}$ as a cone over $A(K)$ and using the natural cone structure of Cone $(\Delta(K))$.

Corollary 3. Let $F: \Delta(L) \rightarrow I^{L}$ be a correspondence having the following properties:

a) $F^{-1}: \operatorname{im}(F) \rightarrow \Delta(L)$ is acyclic-valued, 
b) $\operatorname{im}(F)$ misses the set $A(L) \cup\left\{v_{+}\right\}$, and

c) we have $F^{-1}\left(B\left(L_{0}\right)\right) \subset \Delta\left(L_{0}\right)$ for every set $L_{0} \subset L$.

Then in order for $\operatorname{im}(F)$ to separate $I^{L}$ between $A(L)$ and $\left\{v_{+}\right\}$, it is necessary and sufficient that $F \cap\left(\delta \Delta(L) \times \delta I^{L}\right)$ witnessed the property $\mathcal{S}$ for $F$.

Proof. Let $h: I^{L} \rightarrow \operatorname{Cone}(\Delta(L))$ be the homeomorphism defined in the Remark and let $g: \operatorname{Cone}(\Delta(L)) \rightarrow \Delta(L) \times[0,1]$ be a homeomorphism that "flattens" to $\Delta(L) \times$ $\{1\}$ a small neighborhood $U$, in the boundary of the cone, of the vertex point. We request that this neighborhood be so small that it misses the set $\operatorname{im}(h F)$; also, we request that $g^{-1}\left(\Delta\left(L_{0}\right) \times[0,1]\right) \subset B\left(L_{0}\right)$ for each $L_{0} \subset L$. Clearly, $\operatorname{im}(F)$ separates $I^{L}$ between $v_{+}$and $A(L)$ if and only if it separates it between $U$ and $A(L)$ and thus if and only if $\operatorname{im}(g h F)$ separates $\Delta(L) \times[0,1]$ between $\Delta(L) \times\{0\}$ and $\Delta(L) \times\{1\}$. The result now follows from Lemma 4 applied to the correspondence $g h F$.

Remark 3. Taking levelwise convexification produces a correspondence with property a), while preserving properties b) and c). In turn, the saturation $F^{+}$has properties b) and c) whenever $\operatorname{im}(F) \subset \delta I^{L}$.

Lemma 5. Let $F: \Delta(L) \rightarrow I^{L}$ be a saturated correspondence having property $\mathcal{S}$ for $\Delta(L)$. Then, this property is witnessed by $F \cap\left(\delta \Delta(L) \times \delta I^{L}\right)$.

Proof. Let us write $\Delta$ for $\Delta(L)$. It suffices to prove that the homomorphism $\tilde{H}(j)$ induced by the inclusion $j: F \cap\left(\delta \Delta \times \delta I^{L}\right) \hookrightarrow F \mid \delta \Delta$ is surjective. To this end write

$$
\begin{aligned}
Z & :=\left\{\left(p, x_{1}, x_{2}\right): p \in \delta \Delta, x_{1} \in F(p), x_{2} \in x_{1}^{+}(p)\right\}, \\
Z_{0} & :=\left\{\left(p, x_{1}, x_{2}\right) \in Z: x_{2} \in \delta I^{L}\right\}
\end{aligned}
$$

where by $x_{1}^{+}(p)$ we denote the value at $p$ of the saturation of the constant correspondence taking value $\left\{x_{1}\right\}$. Also, let $\pi_{i}: Z_{0} \rightarrow F \mid \delta \Delta$ be defined by $\pi_{i}\left(p, x_{1}, x_{2}\right)=$ $\left(p, x_{i}\right)$ for $i=1,2$. Since $x_{1}^{+}(p) \subset F(p)$ for $x_{1} \in F(p)$ and the set $x_{1}^{+}(p)$ is convex, the map $\pi_{2}$ does indeed take values in $F \mid \delta \Delta$, as does the homotopy between $\pi_{1}$ and $\pi_{2}$ defined by $h_{t}\left(p, x_{1}, x_{2}\right)=\left(p, t x_{1}+(1-t) x_{2}\right)$.

Moreover, the map $\pi_{1}$ is acyclic and surjective, since each cone $x_{1}^{+}(p)$ intersects $\delta I^{L}$ along an acyclic set. By Vietoris' theorem, $\tilde{H}\left(\pi_{1}\right)$ is an isomorphism. Since $\operatorname{im}\left(\tilde{H}\left(\pi_{1}\right)\right)=\operatorname{im}\left(\tilde{H}\left(\pi_{2}\right)\right) \subset \operatorname{im}(\tilde{H}(j))$, the conclusion follows.

The following property of separators enables one to apply the established results:

Lemma 6. For $i=1, \ldots, n$, let $B_{i}, S_{i}, U_{i}$ be closed subsets of a cube $X$ such that $S_{i}$ separates $X$ between $B_{i}$ and $X \backslash U_{i}$. Then $\bigcup_{i=1}^{n} S_{i} \cap \bigcap_{i=1}^{n} U_{i}$ separates $X$ between $\bigcap_{i=1}^{n} B_{i}$ and $X \backslash \bigcap_{i=1}^{n} U_{i}$.

Proof. By induction on $n$, it suffices to prove the result for $n=2$. Let $V_{i}$ denote the union of those components of $X \backslash S_{i}$ that intersect $B_{i}$. Then $B_{i} \subset V_{i} \subset U_{i}$ and $\delta V_{i} \subset S_{i}$. Hence the neighborhood $V:=V_{1} \cap V_{2}$ of $B_{1} \cap B_{2}$ misses $X \backslash U_{1} \cap U_{2}$ and $\delta V \subset\left(\delta V_{1} \cap \overline{V_{2}}\right) \cup\left(\overline{V_{1}} \cap \delta V_{2}\right) \subset\left(S_{1} \cap U_{2}\right) \cup\left(U_{1} \cap S_{2}\right)=\left(S_{1} \cup S_{2}\right) \cap U_{1} \cap U_{2}$.

Proof of Theorem 2. We temporarily fix an integer $n$ and denote by $J$ the segment $\left[a-\frac{1}{n}, b+\frac{1}{n}\right]$. For $L \in \mathcal{L}$ and $p \in \Delta(L) \subset \Delta(K)$, let $F_{L}^{\prime}(p)=\left\{y \in J^{K}: y^{L} \in\right.$ $\left.c\left(F_{L}^{+}\right)(p)\right\}$, where ${ }^{+}$denotes saturating a correspondence with respect to $J^{L}$. The correspondence $c\left(F_{L}^{+}\right): \Delta(L) \rightarrow J^{L}$ satisfies all the conditions a), b) and c) from Corollary 3 and $c\left(F_{L}^{+}\right) \cap\left(\delta \Delta(L) \times \delta J^{L}\right)$ witnesses the property $\mathcal{S}$ of $c\left(F_{L}^{+}\right)$for 
$\Delta(L)$ (since by Lemma 5 this is so with $c\left(F_{L}^{+}\right)$replaced by its subset $F_{L}^{+}$). With $v_{+}=\left(b+\frac{1}{n}, \ldots, b+\frac{1}{n}\right)$ it therefore follows easily from Corollary 3 that

(6) the set $\operatorname{im}\left(F_{L}^{\prime}\right)$ separates $J^{K}$ between $v_{+}$

$$
\text { and } A^{\prime}(L):=\left\{y \in J^{K}: y^{L} \in A(L)\right\} \text {. }
$$

Next, for $L \in \mathcal{L}$ we denote by $U_{L}^{\prime}$ the convex hull of $\operatorname{im}\left(F_{L}^{\prime}\right) \cup U_{L} \cup\left\{v_{+}\right\}$. Then the set $I^{K} \backslash U_{L}^{\prime}$ is connected and contains $A^{\prime}(L)$, while being disjoint from $\left\{v_{+}\right\} \cup \operatorname{im}\left(F_{L}^{\prime}\right)$. It follows thus from (6) that $\operatorname{im}\left(F_{L}^{\prime}\right)$ separates $v_{+}$from $J^{K} \backslash U_{L}^{\prime}$, and from Lemma 6 that the set

$$
S:=\bigcup_{L \in \mathcal{L}} \operatorname{im}\left(F_{L}^{\prime}\right) \cap \bigcap_{L \in \mathcal{L}} U_{L}^{\prime}
$$

separates $v_{+}$from $J^{K} \backslash \bigcap_{L \in \mathcal{L}} U_{L}^{\prime}$ and hence also from $A:=\bigcup_{L \in \mathcal{L}} A^{\prime}(L)$ (all separations in $\left.J^{K}\right)$.

Since $\bigcup \mathcal{L}=K$ we have $A=A(K)$ and consequently $A(K)$ is separated from $v_{+}$ by $S$.

Let $G$ be the correspondence $\bigcup_{L \in \mathcal{L}} F_{L}^{\prime} \cap \bigcap_{L \in \mathcal{L}} \Delta(K) \times U_{L}^{\prime}$. Then $\operatorname{im}(c G) \supset$ $\operatorname{im}(G)=S$ and $c G$ satisfies the assumptions of Corollary 3, with $K$ in place of $L$. Thus, applying this corollary again, we infer that $c G$ has property $\mathcal{S}$ for $\Delta(K)$.

Finally, we allow $n$ to vary and thus for each $n$ produce a correspondence $G_{n}$ : $\Delta(K) \rightarrow \mathbb{R}^{K}$ such that $c\left(G_{n}\right)$ has property $\mathcal{S}$ for $\Delta(K)$ and $\Gamma=\bigcap_{n} G_{n}$. Then, $c \Gamma=\bigcap_{n} c G_{n}$ and it remains to apply Lemma 2(b).

The above reasoning also establishes the following (we skip the details):

Proposition 1. Let a closed segment $I$ be contained in the interior of another closed segment $J=[a, b]$, and let $F: \Delta(K) \rightarrow I^{K}$ be a saturated correspondence such that the set $F^{-1}(y)$ is acyclic for every $y \in \operatorname{im}(F)$. Then, the correspondence $F$ has property $\mathcal{S}$ for $\Delta(K)$ iff the image of its $J^{K}$-saturation separates the cube $J^{K}$ between the vertex $v_{+}=(b, \ldots, b)$ and the union of all the faces of $J^{K}$ disjoint from $v_{+}$.

\section{Games of incomplete information on ONE Side}

3.1. The model. A brief description of the games follows. There are two players, Player One and Player Two. There is a finite set $K$ of states of nature. Nature chooses a state $k \in K$ according to a commonly known probability $p_{0} \in \Delta(K)$, and the first player, but not the second player, is informed concerning nature's choice. The first player and second player have sets of moves $I$ and $J$, respectively. The play is repeated indefinitely, with the chosen state remaining constant.

Two independent aspects of the play need explanation, the payoffs and the information structure.

Let $A^{k}$ and $B^{k}$ for all $k \in K$ be the corresponding $|I| \times|J|$ payoff matrices for Players One and Two, respectively. At each stage of play, $A^{k}(i, j)$ and $B^{k}(i, j)$ are the payoffs to Player One and Player Two, respectively, when Player One chooses $i \in I$, Player Two chooses $j \in J$ and the state of nature is $k \in K$. We assume, without loss of generality, that all payoffs for Player One are positive, meaning that every entry $A^{k}(i, j)$ will be a positive number.

There are two sets of finite signals $R$ and $S$, received by the first and second players, respectively. There is a stochastic function, $\Lambda: K \times I \times J \rightarrow \Delta(R \times S)$. 
After each stage in which $i \in I$ and $j \in J$ were played and $k$ is the state of nature, a member $(r, s)$ of $R \times S$ is determined by $\Lambda(k, i, j) ; r$ is communicated to Player One, and $s$ is communicated to Player Two.

A behavior strategy of Player One is an infinite sequence $\alpha=\left(\alpha_{1}, \alpha_{2}, \ldots\right)$ such that for each $l, \alpha_{l}$ is a mapping from $K \times(I \times R)^{l-1}$ to $\Delta(I)$. A behavior strategy of Player Two is an infinite sequence $\beta=\left(\beta_{1}, \beta_{2}, \ldots\right)$ such that for each $l, \beta_{l}$ is a mapping from $(J \times S)^{l-1}$ to $\Delta(I)$. (The inclusion of $I^{l-1}$ and $J^{l-1}$ in the definition of behavior strategies for players One and Two, respectively, reflects the important assumption, used later, of perfect recall of one's own past moves.) Let $\mathcal{I}$ and $\mathcal{J}$ be the set of behavior strategies of Players One and Two, respectively.

Define the set of finite play histories of length $l$ to be $\mathcal{H}_{l}:=K \times(I \times J)^{l}$, and define $\mathcal{H}_{l}^{k}$ to be the subset $\{k\} \times(I \times J)^{l}$.

For every $k \in K$ and $h=\left(k, i_{1}, j_{1}, \ldots, i_{l}, j_{l}\right) \in \mathcal{H}_{l}^{k} \subset \mathcal{H}_{l}$, define

$$
f_{l}(h)=\frac{1}{l} \sum_{m=1}^{l} A^{k}\left(i_{m}, j_{m}\right) \quad \text { and } \quad g_{l}(h)=\frac{1}{l} \sum_{m=1}^{l} B^{k}\left(i_{m}, j_{m}\right) .
$$

For any fixed $k \in K$, every pair of behavior strategies $\alpha \in \mathcal{I}$ and $\beta \in \mathcal{J}$ induces a probability measure $\mu_{\alpha, \beta}^{l, k}$ on $\mathcal{H}_{l}^{k}$. With the initial probability $p_{0}$, such a pair induces also a probability measure $\mu_{\alpha, \beta}^{l}$ on $\mathcal{H}_{l}$. A uniform (Nash) equilibrium (see Renault [Re], also [AM], $[\mathrm{So}]$ and [MSZ]) is a pair of behavior strategies $\alpha \in \mathcal{I}$ and $\beta \in \mathcal{J}$ such that for every $k \in K, a^{k}=\lim _{m \rightarrow \infty} \int_{\mathcal{H}_{m}^{k}} f_{m}(h) d \mu_{\alpha, \beta}^{m, k}$ and $b^{k}=$ $\lim _{m \rightarrow \infty} \int_{\mathcal{H}_{m}^{k}} g_{m}(h) d \mu_{\alpha, \beta}^{m, k}$ exist and, for every pair $\alpha^{*} \in \mathcal{I}$ and $\beta^{*} \in \mathcal{J}$,

$$
\lim _{m \rightarrow \infty} \sup \int_{\mathcal{H}_{m}} f_{m}(h) d \mu_{\alpha^{*}, \beta}^{m} \leq \sum_{k} p_{0}^{k} a^{k}, \quad \lim _{m \rightarrow \infty} \sup \int_{\mathcal{H}_{m}} g_{m}(h) d \mu_{\alpha, \beta^{*}}^{m} \leq \sum_{k} p_{0}^{k} b^{k} .
$$

3.2. Nonrevealing strategies. For every $j \in J$, let $\Lambda^{j}:(K \times I) \rightarrow \Delta(S)$ be defined so that $\Lambda^{j}(k, i)$ is the marginal probability on the signals $S$ determined by the moves $i \in I, j \in J$ and the state $k \in K$. Define $\bar{\Lambda}^{j}: \Delta(I)^{K} \rightarrow \Delta(S)^{K}$ so that for every $\sigma \in \Delta(I)^{K}$ with $\sigma=\left(\sigma^{k} \mid k \in K\right), \bar{\Lambda}^{j}(\sigma)$ is the $|K|$-tuple of probabilities on $S$ determined by $\bar{\Lambda}^{j}(\sigma)^{k}:=\sum_{i \in I} \sigma_{i}^{k} \Lambda^{j}(k, i)$. For every $L \subset K$, define

$$
N R(L):=\left\{\sigma \in \Delta(I)^{K} \mid \forall j, \text { for every pair } k, k^{\prime} \in L, \quad \bar{\Lambda}^{j}(\sigma)^{k}=\bar{\Lambda}^{j}(\sigma)^{k^{\prime}}\right\} .
$$

By the linearity of the functions $\bar{\Lambda}^{j}$, for every $L$, as long as it is nonempty, $N R(L)$ is a convex polytope.

For every $p \in \Delta(K)$, define $\operatorname{supp}(p):=\left\{k \in K \mid p^{k}>0\right\} \subset K$.

For every $\sigma \in \Delta(I)^{K}$ and $\tau \in \Delta(J)$, define $\sigma A \tau \in \mathbb{R}^{K}$ (respectively $\sigma B \tau \in \mathbb{R}^{K}$ ) as $(\sigma A \tau)^{k}:=\sigma^{k} A^{k} \tau$ (respectively $\left.(\sigma B \tau)^{k}:=\sigma^{k} B^{k} \tau\right)$.

Define the function $a^{*}$ on $\Delta(K)$ by

$$
a^{*}(p)=\max _{\sigma \in N R(\operatorname{supp}(p))} \min _{\tau \in \Delta(J)} p \cdot \sigma A \tau=\min _{\tau \in \Delta(J)} \max _{\sigma \in N R(\operatorname{supp}(p))} p \cdot \sigma A \tau,
$$

where we set $a(p)=-\infty$ if $N R(\operatorname{supp}(p))=\emptyset$.

The function $a^{*}$ is important because the value of the function $\operatorname{cav}\left(a^{*}\right)$ at $p \in$ $\Delta(K)$ is the value of an infinitely repeated zero-sum game where the payoff matrices of the second player satisfy $B^{k}(i, j)=-A^{k}(i, j)$ for every $i, j$, and $k$; see Aumann and Maschler [AM], Theorem C, p. 191 and Mertens, Sorin, and Zamir [MSZ, Theorem 3.5, p. 231. More particularly, for any $x \in \mathbb{R}^{K}$ such that $x \cdot q \geq a^{*}(q)$ for 
all $q \in \Delta(K)$, there is a behavior strategy $\beta \in \mathcal{J}$ for the second player such that for every behavior strategy $\alpha \in \mathcal{I}$ for the first player and for every $k \in K$,

$$
\lim _{m \rightarrow \infty} \sup \int_{\mathcal{H}_{m}^{k}} f_{m}^{k}(h) d \mu_{\alpha, \beta}^{m, k} \leq x^{k} .
$$

This result, used critically by Renault in the construction of equilibrium behavior strategies, was proven first by E. Kohlberg [Ko] in the context of a deterministic but not necessarily state-independent $\Lambda^{j}$, and in our context by Mertens, Sorin, and Zamir, MSZ, Section 3d, pp. 234-240.

For every $L \subset K$, define $a_{L}^{*}: \Delta(L) \rightarrow \mathbb{R}$ by

$$
a_{L}^{*}(p)=\max _{\sigma \in N R(L)} \min _{\tau \in \Delta(J)} p \cdot \sigma A \tau=\min _{\tau \in \Delta(J)} \max _{\sigma \in N R(L)} p \cdot \sigma A \tau .
$$

Clearly, $a^{*}=\max _{L \subset K} a_{L}^{*}$ and whenever $N R(L)$ is not empty, then $a_{L}^{*}$ is Lipschitz.

Define a subset $L \subset K$ to be extendable to $N \supset L$ if $N R(L) \neq \emptyset$ and every $\sigma \in N R(L)$ can be replaced by a $\bar{\sigma} \in N R(N)$ such that $\sigma^{k}=\bar{\sigma}^{k}$ for every $k \in L$. Define a nonempty subset $L \subset K$ to be nonextendable if there exists no properly containing $N \supset L$ with $L$ extendable to $N$. Define $\mathcal{F}$ to be the family of nonextendable subsets of $K$. Since $N R(\{k\})$ is always all of $\Delta(I)^{K}$ for any $k \in K$ and an $L \subset K$ maximal with the property $N R(L) \neq \emptyset$ will be in $\mathcal{F}$, we know that $\bigcup_{L \in \mathcal{F}} L=K$.

We define an $L \in \mathcal{F}$ to be singular if for all $j \in J, \bar{\Lambda}^{j}(N R(L))$ is a singleton. We say that Player One can send nonrevealing signals if $K \in \mathcal{F}$ and $K$ is not singular. We will prove the existence of equilibria with a weaker assumption, namely, that every member of $\max \mathcal{F}$ is not singular, which is equivalent to the nonsingularity of all members of $\mathcal{F}$, since only maximal members of $\mathcal{F}$ can be singular.

For any nonempty $N \subset K$, define $F(N):=\bigcap_{N \subset L \in \mathcal{F}} L$ for all $N \subset K$, with $F(N)=K$ in the case of an empty intersection.

Lemma 7. $\mathcal{F}$ is closed under nonempty intersection and, for all nonempty $N \subset K$ with $N R(N) \neq \emptyset, F(N)=\{k \mid \forall \sigma \in N R(N)$ there exists a $\hat{\sigma} \in N R(N \cup\{k\})$ such that for every $\left.n \in N, \sigma^{n}=\hat{\sigma}^{n}\right\}$.

Proof. Let us assume that $L_{1}$ and $L_{2}$ are in $\mathcal{F}$. Suppose for the sake of contradiction that $L_{1} \cap L_{2}$ can be extended to $N \supset L_{1} \cap L_{2}$. By symmetry, assume $N \not \subset L_{1}$. Since $L_{1}$ is not extendable, there is a $j \in J$ and a strategy $\sigma \in N R\left(L_{1}\right)$ such that there is no $\bar{\sigma} \in N R\left(N \cup L_{1}\right)$ with $\bar{\sigma}^{k}=\sigma^{k}$ for every $k \in L_{1}$. Notice that $N R\left(L_{1}\right) \subset N R\left(L_{1} \cap L_{2}\right)$. If there were a $\hat{\sigma} \in N R(N)$ with $\hat{\sigma}^{k}=\sigma^{k}$ for every $k \in L_{1} \cap L_{2}$, consider $\bar{\sigma}$ defined by $\bar{\sigma}^{k}:=\sigma^{k}$ if $k \in L_{1}$ and $\bar{\sigma}^{k}:=\hat{\sigma}^{k}$ if $k \notin L_{1}$. For any $j \in J$, consider $r^{j} \in \Delta(S)$ to be $\bar{\Lambda}^{j}(\hat{\sigma})^{k}$ for all $k \in N$. Since $\emptyset \neq L_{1} \cap L_{2} \subset N$, $r^{j}$ is $\bar{\Lambda}^{j}(\sigma)^{k}$ or $\bar{\Lambda}^{j}(\hat{\sigma})^{k}$ for all $k \in L_{1} \cap L_{2}, r^{j}$ is $\bar{\Lambda}^{j}(\hat{\sigma})^{k}$ for $k \in N$, and $r^{j}$ is $\bar{\Lambda}^{j}(\sigma)^{k}$ for $k \in L_{1}$. Therefore $r^{j}$ is also $\bar{\Lambda}^{j}(\bar{\sigma})^{k}$ for all $k \in L_{1} \cup N$, a contradiction.

The set $Q:=\{k \mid \forall \sigma \in N R(N)$ there exists a $\hat{\sigma} \in N R(N \cup\{k\})$ such that for every $\left.n \in N, \sigma^{n}=\hat{\sigma}^{n}\right\}$ is nonextendable, since if $Q$ were extendable to some $Q^{\prime}$ strictly containing $Q$, then choosing any $k \in Q^{\prime} \backslash Q$ we would have to conclude that $k$ was already in $Q$, a contradiction. Furthermore, if $P$ is a set between $N$ and $Q$, $N R(P) \subset N R(N)$ would imply that $P$ is extendable to $Q$. So from the above we conclude that $Q=F(N)$.

Lemma 8. $a^{*}=\max _{L \in \mathcal{F}}\left(a_{L}^{*}\right)$ holds. 
Proof. If $N R(L) \neq \emptyset$, then Lemma 7 shows that $L$ is extendable to $F(L)$, meaning that any strategy in $N R(L)$ can be replaced by an equivalent one in $N R(F(L))$. This means that $a_{L}^{*}$ is not greater than $a_{F(L)}^{*}$ on $\Delta(L)$.

3.3. Joint plans. Usually, a joint plan is defined to be a kind of contract between the players concerning their behavior. Due to the complexity of the contractual behavior in this paper needed to establish an equilibrium, we will define a joint plan to be only the underlying probability and payoff structure.

The main component of a joint plan is what we define to be a "proto-joint-plan" for some $p \in \Delta(K)$. A proto-joint-plan is a pair of strategies in $\Delta(I)^{K}$ and $\Delta(J)$ defining the normal behavior of the players at some subset of the stages of play. A "nonrevealing joint plan" for some $p \in \Delta(K)$ is a mixture of proto-joint-plans for the same $p \in \Delta(K)$, and defines the average payoffs of the players in the limit of play. A "joint plan" for some $p \in \Delta(K)$ is a set $V \subset \Delta(K)$ whose convex hull contains $p$ along with a nonrevealing joint plan for every $p \in V$.

For every $p \in \Delta(K)$, define $F(p):=F(\operatorname{supp}(p))$.

For every $L \subset K$, define the equivalence relation $\sim_{L}$ on $N R(L)$ by $\sigma \sim_{L} \hat{\sigma}$ if and only if $\bar{\Lambda}^{j}(\sigma)^{k}=\bar{\Lambda}^{j}(\hat{\sigma})^{k}$ for all $j \in J$ and $k \in L$. A proto-joint-plan for some $p \in \Delta(K)$ is a pair $\sigma \in \Delta(I)^{K}, \tau \in \Delta(J)$ such that $\sigma \in N R(F(p))$ and for every $k \in F(p)$,

$$
\sigma^{k} A^{k} \tau=\max _{\bar{\sigma} \sim F(p)} \bar{\sigma}^{k} A^{k} \tau
$$

The $\mathbb{R}^{K}$ Player One payoff corresponding to the proto-joint-plan is the vector $x$ in $\mathbb{R}^{K}$ defined by $x^{k}=\sigma^{k} A^{k} \tau$. A proto-joint-plan is two-best-reply (see [Re]) if $p \cdot \sigma B \tau=\max _{\bar{\tau} \in \Delta(J)} p \cdot \sigma B \bar{\tau}$. For any $L \supset F(p)$, an $L$-pure proto-joint-plan for $p$ is a pair $\sigma \in N R(L)$ and $\tau \in \Delta(J)$ such that $\sigma^{k} A^{k} \tau=\max _{\bar{\sigma} \sim_{L} \sigma} \bar{\sigma}^{k} A^{k} \tau$ for every $k \in L$.

Remark 4. An $L$-pure proto-joint-plan is also a proto-joint-plan. The fact that $N R(L) \subset N R(F(p))$ follows from $F(p) \subset L$. Now we need to show that the payoff maximality condition in the definition of $L$-purity implies the payoff maximality condition in the definition of a proto-joint-plan. Assuming that $\sigma \in N R(L) \subset$ $N R(F(p))$ and $\sigma$ satisfies $\sigma^{k} A^{k} \tau=\max _{\bar{\sigma} \sim_{L} \sigma} \bar{\sigma}^{k} A^{k} \tau$ for every $k \in L$, let $\hat{\sigma}$ be any other member of $N R(F(p))$ with $\hat{\sigma} \sim_{F(p)} \sigma$. We can define, as in the proof of Lemma 7, a $\bar{\sigma} \in N R(L)$ by $\bar{\sigma}^{k}=\sigma^{k}$ if $k \notin F(p)$ and $\bar{\sigma}^{k}=\hat{\sigma}^{k}$ if $k \in F(p)$, with $\bar{\sigma}^{k} A^{k} \tau=\hat{\sigma}^{k} A^{k} \tau$ if $k \in F(p)$ and $\bar{\sigma} \sim_{L} \sigma$.

A nonrevealing joint plan for $p \in \Delta(K)$ is a finite convex combination of proto-joint-plans for the same $p$. It is two-best-reply if every proto-joint-plan in the convex combination is two-best-reply. Given that $y \in \mathbb{R}^{K}$ is the appropriate convex combination of the Player One payoffs from proto-joint-plans corresponding to the nonrevealing joint plan, a Player One payoff of the nonrevealing joint plan is any $\bar{y} \in[0, \mathbb{R}]^{K}$ such that $\bar{y}^{k}=y^{k}$ if $p^{k}>0$ and $\bar{y}^{k} \geq y^{k}$ if $k \in F(p)$. Notice that the payoff of a proto-joint-plan for $p \in \Delta(K)$ is defined with respect to all of $K$, while the payoffs of a nonrevealing joint plan are defined essentially with respect to $F(p)$ and allow for increasing the values for coordinates outside of $\operatorname{supp}(p)$. The reason for the more restrictive definition for proto-joint-plans is to accommodate the property of purity when dealing with an $L \in \mathcal{F}$ properly containing $F(p)$.

A joint plan for $p \in \Delta(K)$ is 
1) a finite set $V \subset \Delta(K)$ such that $p \in \operatorname{conv}(V)$,

2) for every singular $L \in \max \mathcal{F}$ at most one member $v$ of $V$ with $F(v)=L$,

3) for every $v \in V$ an associated nonrevealing joint plan for $v$.

A joint plan is two-best-reply if every nonrevealing joint plan associated with the joint plan is two-best-reply. The joint plan for $p$ is balanced if there exists an $x \in \mathbb{R}^{K}$ such that for every $v \in V, x$ is a Player One payoff of the nonrevealing joint plan associated with $v$, and such an $x \in \mathbb{R}^{K}$ is called a Player One payoff for the balanced joint plan. Furthermore, if $N \supset F(p)$ and an $x \in \mathbb{R}^{K}$ is a Player One payoff for the balanced joint plan, then we will also call $x^{N}$ a Player One payoff for the balanced joint plan.

Remark 5. Originally for the case of standard information, a "joint plan" was defined using members of $\Delta(I \times J)$, [AM]. Because every member of $\Delta(I \times J)$ can be represented as a convex combination of members of $\Delta(I) \times \Delta(J)$, our definition in the context of the standard information case does not differ essentially from the original definition.

We fix a number $M$ such that $M>\max _{i, j, k} A^{k}(i, j)$ and, for every $L \in \mathcal{F}$ and $p \in \Delta(L)$, we let the correspondence $\Psi_{L}: \Delta(L) \rightarrow[0, M]^{L}$ be defined by $\Psi_{L}(p)$ being the $\mathbb{R}^{L}$ coordinates of the Player One payoffs of two-best-reply proto-jointplans that are $L$-pure. Renault $[\mathrm{Re}$. proved the following lemma, although only explicitly in the case of state-independent signalling. For the sake of completeness, we repeat his proof.

Lemma 9 (J. Renault [Re]). For every $L \in \mathcal{F}, \Psi_{L}: \Delta(L) \rightarrow[0, M]^{L}$ is an uppersemi-continuous correspondence with the property that, for every $p, q \in \Delta(L)$, there is an $x \in \Psi_{L}(p)$ such that $x \cdot q \geq a_{L}^{*}(q)$.

Proof. First we show that $\Psi_{L}$ satisfies the latter property. Given $p, q \in \Delta(L)$, let $\sigma \in N R(L)$ and $\tau \in \Delta(J)$ be a Nash equilibrium in the one-shot game defined by the convex polytopal strategy sets $N R(L)$ and $\Delta(J)$ where $\sigma$ and $\tau$ give a payoff of $q \cdot \sigma A \tau$ to the first player and $p \cdot \sigma B \tau$ to the second player. Because $a_{L}^{*}$ is defined using $N R(L)$ and $\Delta(J)$, such a Nash equilibrium attains a payoff of at least $a_{L}^{*}(q)$ for Player One. By the Nash equilibrium property, for any $k \in \operatorname{supp}(q), \sigma^{k} A^{k} \tau$ cannot be improved upon by another $\hat{\sigma}$ with $\hat{\sigma} \sim_{L} \sigma$. If $k \notin \operatorname{supp}(q)$, then we choose $\sigma^{k}$ so as to attain the maximum of $\left\{\bar{\sigma}^{k} A^{k} \tau \mid \bar{\sigma} \sim_{L} \sigma\right\}$. We can make this choice freely without upsetting the Nash equilibrium property because $k \notin \operatorname{supp}(q)$. Likewise, the Nash equilibrium property gives the two-best-reply property.

We assume there are sequences $\sigma_{l} \rightarrow \sigma, \tau_{l} \rightarrow \tau$, and $p_{l} \rightarrow p$ such that for every $l$, $\sigma_{l}$ and $\tau_{l}$ are two-best-reply proto-joint-plans for $p_{l}$ that are $L$-pure. It suffices to show that $\sigma$ and $\tau$ is a two-best-reply proto-joint-plan for $p$ that is $L$-pure. Showing the two-best-reply property and that $\sigma$ is in $N R(L)$ are straightforward. It is left to show that if there were a $\hat{\sigma} \in N R(L)$ with $\sigma \sim_{L} \hat{\sigma}$ for some $k \in L$ and $\hat{\sigma}^{k} A^{k} \tau>\sigma^{k} A^{k} \tau$, then the same would occur for some $\sigma_{l}^{k}$ and $\tau_{l}$ close enough to $\sigma^{k}$ and $\tau$. We consider the directional vector $w:=\hat{\sigma}^{k}-\sigma^{k}$. Any fraction $0<t<1$ of $w$ added to the $\sigma_{l}^{k}$ would generate for large enough $l$ the same distribution on signals for every $j \in J$ and also yield an increase in payoff to the first player at the state $k$. (A fraction $0<p<1$ must be used to avoid having $\sigma_{l}^{k}+t w$ outside of $\Delta(I)$ for all $l$.)

For $N \supset L$, a vector $x \in \mathbb{R}^{N}$ dominates a function $f: \Delta(L) \rightarrow \mathbb{R}$ if $x \mid \Delta(L) \geq f$ or, equivalently, if $x \cdot q \geq f(q)$ for all $q \in \Delta(L)$. 
From Lemma 9, Renault $[\mathrm{Re}$ proved essentially something more, namely that for every $p$ in the interior of $\Delta(L)$, there exists a balanced two-best-reply joint plan with a payoff vector for Player One that dominates $a_{L}^{*}$.

Theorem 3. Given that every member of $\max \mathcal{F}$ is nonsingular, for every $p_{0} \in$ $\Delta(K)$ there exists a balanced two-best-reply joint plan such that a payoff vector of Player One dominates $a^{*}$.

Proof. For every $L \in \mathcal{F}$, define $\tilde{\Psi}_{L}: \Delta(L) \rightarrow[0, M]^{L}$ by $\tilde{\Psi}_{L}(p):=\operatorname{conv}\left(\Psi_{L}(p)\right)$. From Carathéodory's theorem and Lemma 9 , we know that $\tilde{\Psi}_{L}$ is upper-semicontinuous and, also by Lemma 9, it has the property that for every $p, q \in \Delta(L)$, there is an $x \in \tilde{\Psi}_{L}(p)$ such that $x \cdot q \geq a_{L}^{*}(q)$. Let $\tilde{\Psi}_{L}^{+}$be the $[0, M]^{L}$ saturation of $\tilde{\Psi}_{L} ; \tilde{\Psi}_{L}^{+}(p)$ is a subset of the two-best-reply nonrevealing joint plan payoffs in $[0, M]^{L}$. For every $L \in \mathcal{F}$ define $F_{L}: \Delta(L) \rightarrow[0, M]^{L}$ by $F_{L}:=c\left(\tilde{\Psi}_{L}^{+}\right) \cap(\Delta(L) \times\{y \in$ $\left.\left.[0, M]^{L}: y \mid \Delta(L) \geq a_{L}^{*}\right\}\right)$. From Theorem 1 it follows that $F_{L}$ has property $\mathcal{S}$. By the fact that all members of $\mathcal{F}$ are nonsingular, and therefore there is no restriction on the forming of convex combinations in $\Delta(L)$ to generate joint plans, we have for every $p \in \Delta(L)$ that $F_{L}(p)$ is a subset of the $\mathbb{R}^{L}$ vectors that dominate $a_{L}^{*}$ and are Player One payoffs of balanced two-best-reply joint plans for $p$.

Set $U_{L}:=\left\{y \in[0, M]^{K}: y^{L} \mid \Delta(L) \geq a_{L}^{*}\right\}$. We know from Corollary 2 that for every $p_{0} \in \Delta(K)$, we have a finite number of sets $L_{1}, \ldots, L_{s} \in \mathcal{F}$, points $p_{i} \in \Delta\left(L_{i}\right) \subset \Delta(K)$ for $1 \leq i \leq s$, and positive numbers $t_{1}, \ldots, t_{s}$ with $\sum_{i=1}^{s} t_{i}=1$, $\sum_{i} t_{i} p_{i}=p$ and a point $x \in[0, M]^{K}$ dominating $a_{L}^{*}$ for all $L \in \mathcal{F}$ such that $x^{L_{i}} \in F_{L_{i}}\left(p_{i}\right)$ for every $i=1, \ldots, s$.

For the conclusion of Theorem 3 , it suffices to show that such an $x \in \mathbb{R}^{K}$ dominates $a^{*}$ and is a Player One payoff of a balanced two-best-reply joint plan for $p_{0}$.

By Lemma $8, x \in \mathbb{R}^{K}$ dominates $a^{*}$. For every $1 \leq i \leq s$, there is a finite set $V_{i} \subset \Delta\left(L_{i}\right)$ such that $p_{i}$ is in the convex hull of $V_{i}$ and for every $p \in V_{i}$, there is a two-best-reply nonrevealing joint plan for $p$ with a payoff of $x$ for the first player. Consider the union $\bigcup_{i} V_{i}$; its convex hull contains $p_{0}$. The fact that no member of $\mathcal{F}$ is singular allows the freedom to take any convex combination in $\Delta(K)$ to form a joint plan, and therefore these two-best-reply nonrevealing joint plans giving the same payoff of $x \in R^{K}$ to the first player satisfy all the conditions to define a two-best-reply balanced joint plan for $p_{0}$.

3.4. From joint plan to equilibrium. In this section we show that a two-bestreply balanced joint plan with a payoff dominating $a^{*}$ generates a uniform equilibrium of the game. We present a summary of the construction of strategies from such joint plans in the case of a state-independent information structure, done by Renault [Re], and then show that a minimal revision of Renault's construction generates uniform equilibrium strategies in our context of state-dependent information.

Let us first index the joint plan by the finite set $V \subset \Delta(K)$ of probabilities corresponding to the nonrevealing joint plans, such that the initial probability $p_{0}$ is in the convex hull of $V$. For every $v \in V$, we have another index set $T_{v}$ for the proto-joint-plans. Let $q^{v, t}$ be the proportion given to the proto-joint-plan indexed by $t \in T_{v}$, with $\sigma_{v, t} \in N R(F(v))$ and $\tau_{v, t} \in \Delta(J)$ the corresponding one-shot mixed strategies. For both index sets, by Carathéodory's theorem, we can assume a finite number of members, no more than $|K|$ for $V$ and no more than $|I||K|+|J|$ for every $T_{v}$. We assume that $x \in \mathbb{R}^{K}$ is the payoff of the joint plan. The pair of 
behavior strategies that Renault constructs we will call the suggested behavior strategies. Renault's suggested behavior strategies will incorporate four aspects:

1) a process by which Player One chooses by lottery a nonrevealing joint plan $v \in$ $V$ and sends to Player Two a noisy signal with the intention of communicating the chosen $v$, and an interpretation of a $v^{\prime} \in V$ by Player Two;

2) a punishment strategy by the second player against the first player, holding him down in payoff to $x^{k}$ or less for every $k$;

3) playing phases, where play should be determined by the chosen nonrevealing joint plan, with proto-joint-plans assigned to subsequences of the playing stages;

4) a mechanism for the second player to monitor the actions of the first player in the playing phases and to determine when to replace his adherence to the interpreted nonrevealing joint plan by punishment in some subsequent playing phases.

For our purposes, we need only to add a fifth aspect to Renault's scheme:

5) a mechanism for every $L \in \mathcal{F}$, given that the chosen state of nature is in $L$, for the first player to confirm (in a way nonrevealing with respect to $L$ ) to the second player that indeed the state of nature is in $L$.

The two-best-reply property makes unnecessary a process by which Player One monitors and punishes Player Two for deviation.

Now we describe Renault's approach in more detail. The game is broken into blocks of stages for signaling and blocks of stages for playing, with alternation between signaling and playing.

In the signaling blocks, $C_{1}, C_{2}, \ldots$, Player One attempts to send a message that means that they should play a nonrevealing joint plan associated to one of the members of $V$. In Renault's context of state-independence, there is only one member in $\mathcal{F}$, namely $K$, so that $a^{*}=a_{K}^{*}$. If $K$ is singular, then $a^{*}$ is concave, and equilibrium existence follows easily. Therefore, Renault could assume that $K$ was nonsingular and that there is at least a line segment $\overline{a, b}$ in $\bar{\Lambda}^{j}(N R(K))$ for some $j \in J$. Let $m$ be large enough such that $2^{m}$ is at least as large as the cardinality of $V$, so that there is a surjective map from $\{a, b\}^{m}$ to $V$. We will refer to a member of $\{a, b\}^{m}$ as a noisy signal, because it is a signal that must be interpreted by Player Two; due to stochastic noise, the signal could be interpreted incorrectly. Player One chooses a member of $V$ according to a state-dependent lottery whose outcome of $v \in V$ means exactly that the signal in $\{a, b\}^{m}$ corresponding to $v$ implies that the posterior probability of $k \in K$ is $v^{k}$. (We can generate the lottery through mixtures of behavior strategies, but with the property of perfect recall concerning one's own moves, by Kuhn's theorem it can also be done with behavior strategies; see [Re and [SST].) The signaling blocks incorporate $m$ sub-blocks of approximately equal length. The purpose of the signaling blocks is to give Player One the opportunity to communicate a member of $\{a, b\}^{m}$, hence also any member of $V$. Player Two interprets that $a$ was intended in the $l$-th coordinate of $\{a, b\}^{m}$ if the average of the signals in $S$ received in the subsequences corresponding to $l$ is closer to $a$ than to $b$, and otherwise he interprets that $b$ was intended. Because the length of the blocks $C_{l}$ grows faster than $l^{2}$, given that Player One is consistent concerning which member in $V$ is to be communicated, with probability one Player Two will receive this message unambiguously. Let $y_{v}$ be the nonrevealing joint payoff payoff associated with $v$. From the equality $y_{v}^{k}=x^{k}$ if $v^{k}>0$ and the 
inequality $y_{v}^{k} \leq x^{k}$ otherwise, we see that the first player is indifferent to which member of $V$ is communicated; (in the state-independent context, $K$ is the only nonextendable set).

The playing blocks alternate with the signaling blocks, but the ratio in length of signaling blocks to playing blocks will approach zero, so that in the limit the signaling blocks are not significant to the payoff. We have for each $v \in V$ a system of mutually disjoint sub-blocks $\left\{B_{l}^{v, t} \mid t \in T_{v}\right\}$ corresponding to the proto-jointplans, such that $\bigcup B_{l}^{v, t}=B_{l}$ and the ratio of $\left|B_{l}^{v, t}\right| / B_{l}$ converges to $q^{v, t}$. With the suggested behavior strategies, if $v$ was chosen by the first player, the first player should play the mixed strategy $\sigma_{v, t} \in \Delta(I)^{K}$ in the blocks $B_{l}^{v, t}$. If $v^{\prime} \in V$ is the message interpreted by the second player and the second player is not currently involved in the process of punishing the first player, he should play $(1-1 / l) \tau_{v^{\prime}, t}+e / l$ in these same blocks, where $e \in \Delta(J)$ is the even distribution on all moves. The even distribution on all moves of Player Two at a rate of $1 / l$ in the $l$-th playing block is a mechanism to randomly check the behavior of the first player, and to do so in a way that does not influence directly the limit average payoff of the game. For each sub-block $B_{l}^{v, t}$ and each $j \in J$ played, the second player compares the observed average distribution of signals in $S$ with the expected distribution $\bar{\Lambda}^{j}\left(\sigma^{v, t}\right)$. If this difference exceeds $1 / l$, then Player Two punishes Player One in sufficiently many following playing blocks so that the length of the punishment dominates the past playing blocks. Player Two does not punish in the signaling blocks, since it is possible that Player Two simply heard the wrong signal and in this case he must give Player One a chance to correct this misinterpretation.

Given that there is a finite stage such that beyond this stage the signals in $\{a, b\}^{m}$ sent and interpreted are the same and all punishment has terminated, we expect with probability one that the suggested behavior strategies will generate a limit average payoff of $x$ to the first player. Given adherence to the suggested behavior strategies, we can expect successful communication of the chosen $v \in V$ with probability one. Furthermore, if Player One adheres to his suggested behavior strategy, Player Two deviations are not profitable due to the two-best-reply condition. To show that the suggested behavior strategies are in equilibrium, Renault showed that

1) with probability one the suggested behavior strategies result in termination of punishment by the second player, and

2) deviations by the first player do not yield to him any average payoff advantage.

This second part is the most involved part of Renault's proof. Because of the maximality condition on Player One's payoff in the definition of a proto-joint-plan, a deviant behavior strategy that would give Player One a higher average payoff must fail to yield the appropriate distribution on the signals in $S$ for those random stages where Player Two is checking. Renault showed that the punishments are sufficiently long and the observational powers of the second player are sufficiently sharp that, in the limit, the first player cannot take any advantage from deviations in the playing blocks that he does not subsequently lose by provoking repetitive punishment.

Our addition. The only addition to Renault's definition of the suggested behavior strategies is in the signaling blocks. We need to add a process of confirmation in 
which the first player guarantees to the second player with probability one that the state of nature is indeed a member of $F(v)$ for the chosen $v \in V$.

Define the set $A_{j}^{k}:=\left\{\left(\bar{\Lambda}^{j}(\sigma)\right)^{k} \mid \sigma \in \Delta(I)^{K}\right\} \subset \Delta(S)$. We recall from the definition of a nonextendable set $L \subset K$ that for every $k \notin L$ there is a $j_{L}^{k} \in J$ and a strategy $\sigma_{L, k} \in N R(L)$ such that the distribution $\chi_{L}^{k} \in \Delta(S)$ defined by $\left.\chi_{L}^{k}:=\bar{\Lambda}^{j_{L}^{k}}\left(\sigma_{L, k}\right)\right)^{k^{\prime}} \in \Delta(S)$ for all $k^{\prime} \in L$ is outside of the set $A_{j_{L}^{k}}^{k}$. Let $\xi^{k}:=$ $\min _{k \notin L \in \mathcal{F}}\left(\right.$ distance $\chi_{L}^{k}, A_{j_{L}^{k}}^{k}$ ). We adjoin to the signaling block, $C_{l},|J||K|$ subblocks, $\tilde{C}_{l}^{j, k}$, of approximately equal length such that the combined length of the $\tilde{C}_{l}^{j, k}$ is approximately equal to the length of $C_{l}$.

Before attempting to communicate the chosen $v \in V$, the first player must first communicate the appropriate member in $\mathcal{F}$, namely $F(v)$. In the $j, k$ sub-blocks $\tilde{C}_{l}^{j, k}$, if $k \notin F(v)$ and $j=j_{F(v)}^{k}$, the first player, with his suggested behavior strategy, should play $\sigma_{F(v), k}$ and otherwise any member of $N R(F(v))$. The second player, with his suggested behavior strategy, should play $j$ in $\tilde{C}_{l}^{j, k}$. Given that Player Two has observed an average of $\alpha^{j, k} \in \Delta(S)$ in the blocks $\tilde{C}_{l}^{j, k}$, we say that the signals received by the second player confirm $L \in \mathcal{F}$ if $L=F(N)$ where $N=\left\{k \in K \mid \forall j \in J\right.$, distance $\left.\left.\left(\alpha^{j, k}, A_{j}^{k}\right) \leq \xi^{k} / 2\right)\right\}$.

For every $L \in \mathcal{F}$, define $V_{L}:=\{v \in V \mid F(v)=L\}$.

Next, in the rest of the $C_{l}$ block, the first player communicates a $v \in V_{L}$ for the appropriate $L \in \mathcal{F}$ exactly as with Renault, given that $L$ is nonsingular with some distinct $a_{L}$ and $b_{L}$ in $\bar{\Lambda}^{j}(N R(L))$ for some $j$. If $L \in \max \mathcal{F}$ is singular, by the definition of a joint plan, there will be only one member $v$ of $V$ satisfying $F(v)=L$, so that the confirmation of $L$ is sufficient for the communication of the appropriate nonrevealing joint plan. Exactly as with Renault, the second player must interpret a member of $v \in V_{L}$ from interpreting a noisy signal in $\left\{a_{L}, b_{L}\right\}^{m}$ for some large enough $m$, where $L$ is the confirmed member of $\mathcal{F}$. If the signals received by the second player in the sub-blocks $\tilde{C}_{l}^{j, k}$ do not confirm any member of $\mathcal{F}$ (because $F(N)=\emptyset$ or $F(N)=K$ and $K \notin \mathcal{F})$ or if $V_{L}=\emptyset$ for the confirmed $L \in \mathcal{F}$, then the second player punishes the first player in the following playing blocks exactly as he would if he were punishing what seemed to be a deviation of play in the $B^{l-1}$ playing block.

With the suggested behavior strategies, since with probability one the intended member of $\mathcal{F}$ along with the intended member of $V$ equals eventually that confirmed and interpreted by Player Two, respectively, with probability one the second player will receive eventually a stable communication of the correct $v \in V$. Due to the definition of $A_{j}^{k}$, Player One has no ability with any positive probability at a state $k$ outside of $F(v)$ to maintain an average signal more than a distance of $\xi^{k} / 2$ outside of $A_{j_{F(v)}^{k}}^{k}$. Therefore, no matter how good for Player One is the convex combination of proto-joint-plan payoffs associated with a $v \in V$ at a state $k \notin F(v)$, Player One has no opportunity to enjoy it at the state $k$ - except in a set of measure zero he cannot get any more than $x^{k}$ by attempting to communicate a $v \in V$ such that $k \notin F(v)$.

\section{REFERENCES}

[AM] Aumann, R. and Maschler, M. (1995). Repeated Games with Incomplete Information. With the collaboration of R. Stearns. M.I.T. Press, Cambridge, MA. MR 96k:90001 
[ES] Eilenberg, S. and Steenrod, N. (1952). Foundations of Algebraic Topology. Princeton University Press, Princeton, N.J. MR 14:398b

[Ko] KohlBerg, E. (1975). "Optimal Strategies in Repeated Games with Incomplete Information." International Journal of Game Theory, 4, 7-24. MR 52:12811

[MSZ] Mertens, J.-F., Sorin, S., and Zamir, S. (1994). Repeated Games, Core Discussion Papers 9420-22, Université Catholique de Louvain.

[Re] Renault, J. (2000). "On Two-player Repeated Games with Lack of Information on One Side and State-independent Signalling", Mathematics of Operations Research, 25, No. 4, pp. 552-572. MR 2002g:91033

[Si] Simon, R. (2002). "Separation of Joint Plan Equilibrium Payoffs From the Min-Max Functions", to appear in Games and Economic Behavior.

[SST] Simon, R., Spież, S. and ToruńczyK, H. (1995). "The Existence of Equilibria in Certain Games, Separation for Families of Convex Functions and a Theorem of Borsuk-Ulam Type," Israel Journal of Mathematics 92, 1-21. MR 97f:90132

[So] Sorin, S. (1983). "Some Results on the Existence of Nash Equilibria for Non-zero-sum Games with Incomplete Information," International Journal of Game Theory 12, 193205. MR 85c:90104

[SZ] Sorin, S. and ZAMIR, S. (1985). "A Two-Person Game with Lack of Information on One and One-Half Sides," Mathematics of Operations Research 10, 17-23. MR 86c:90133

[Sp] Spanier, E. H. (1966). "Algebraic Topology", McGraw-Hill, New York. MR 35:1007

Universität Göttingen, Institut für Mathematische Stochastik, Lotze Strasse 13, 37083 Göttingen, Germany

E-mail address: simon@math.uni-goettingen.de

Institute of Mathematics, Polish Academy of Sciences, Śniadeckich 8, P.O.B. 137, 00-950 WarszaWa, Poland

E-mail address: S.Spiez@impan.gov.pl

Institute of Mathematics, Warsaw University, Banacha 2, 02-097 Warszawa, Poland

E-mail address: H.Torunczyk@impan.gov.pl 